\title{
Charge Transport in Pure and Mixed Phases in Organic Solar Cells
}

Armantas Melianas, Vytenis Pranculis, Donato Spoltore, J ohannes Benduhn, Olle Inganäs, Vidmantas Gulbinas, Koen Vandewal and Martijn Kemerink

The self-archived version of this journal article is available at Linköping University Electronic Press:

http:/ / urn.kb.se/ resolve?urn=urn:nbn:se:liu:diva- 139690

N.B.: When citing this work, cite the original publication.

Melianas, A., Pranculis, V., Spoltore, D., Benduhn, J ., Inganäs, O., Gulbinas, V., Vandewal, K.,

Kemerink, M., (2017), Charge Transport in Pure and Mixed Phases in Organic Solar Cells, Advanced Energy Materials. https:/ / dx.doi.org/ 10.1002/ aenm.201700888

Original publication available at:

https:// dx.doi.org/ 10.1002/ aenm.201700888

Copyright: Wiley: 12 months

http:// eu.wiley.com/WileyCDA/ 


\section{Charge Transport in Pure and Mixed Phases in Organic Solar Cells}

Armantas Melianas*, Vytenis Pranculis, Donato Spoltore, Johannes Benduhn, Olle Inganäs, Vidmantas Gulbinas, Koen Vandewal, Martijn Kemerink*

Armantas Melianas, Prof. Olle Inganäs, Prof. Martijn Kemerink

Department of Physics, Chemistry and Biology, Linköping University, 58183 Linköping, Sweden

Email: ${ }^{*}$ Armantas.Melianas@liu.se

Email: $-\underline{-}$ Martijn.Kemerink@liu.se

Vytenis Pranculis, Prof. Vidmantas Gulbinas

Center for Physical Sciences and Technology, Saulètekio av. 3, LT-02300 Vilnius, Lithuania

Dr. Donato Spoltore, Johannes Benduhn, Prof. Koen Vandewal

Dresden Integrated Center for Applied Physics and Photonic Materials (IAPP) and Institute for Applied Physics, Technische Universität Dresden, 01062 Dresden, Germany

Prof. Vidmantas Gulbinas

Department of General Physics and Spectroscopy, Faculty of Physics, Vilnius University, Saulètekio 9, LT-10222 Vilnius, Lithuania

Keywords: Organic Photovoltaics; Charge Carrier Transport; Tunneling; Low Donor Concentration; Fullerene Domains

\section{Abstract}

In organic solar cells continuous donor and acceptor networks are considered necessary for charge extraction, whereas discontinuous neat phases and molecularly mixed donor-acceptor phases are generally regarded as detrimental. However, the impact of different levels of domain continuity, purity and donor-acceptor mixing on charge transport remain only semi-quantitatively described. Here, we study co-sublimed donor-acceptor mixtures, where the distance between the donor sites is varied in a controlled manner from homogeneously diluted donor sites to a continuous donor network. Using transient measurements, spanning from sub-picoseconds to microseconds we measure photo-generated charge motion in complete photovoltaic devices, to show that even highly diluted donor sites (5.7-10\% molar) in a buckminsterfullerene matrix enable hole transport. Hopping between isolated donor sites occurs by long-range hole tunneling through several buckminsterfullerene molecules ( 4 nm). Hence, 
these results question the relevance of 'pristine' phases and whether a continuous interpenetrating donor-acceptor network is the ideal morphology for charge transport.

\section{Introduction}

Organic photovoltaics (OPV) allows for a low-cost alternative to inorganic solar cells, with recent developments showing power conversion efficiencies of $10-12 \% .{ }^{[1-3]}$ The photoactive layer in an OPV device is most commonly based on a disordered mixture of electron donating and accepting materials - a bulk heterojunction (BHJ). Such interpenetrating donor-acceptor mixtures form complicated multi-length scale morphologies, often involving several phases such as ordered/disordered donor, mixed donor-acceptor and ordered/disordered acceptor. The coexistence of neat and mixed donor-acceptor domains is thought to be beneficial as it could provide an energy cascade ${ }^{[4,5]}$ for charge separation, ${ }^{[6,7]}$ followed by charge transport in the neat phases. However, the neat regions may be discontinuous, forcing charges to cross mixed domains multiple times during extraction. If the mixed regions are severely disordered or contain less than the percolation threshold of required material, charge transport is thought to deteriorate significantly, limiting device performance. ${ }^{[8]}$

The effect of donor-acceptor mixing (or phase purity) on charge transport has been previously addressed using microstructural characterization, ${ }^{[9,10]}$ steady-state $^{[11-13]}$ and/or time-resolved ${ }^{[14-}$ 16] mobility measurements on BHJs with a varying donor-acceptor stoichiometry and/or processing conditions. Although such studies have revealed important trends, they remain mostly semi-quantitative - the question of how pure the neat domains have to be and how detrimental domain discontinuities or donor-acceptor mixing are in relation to charge transport kinetics remains to a large extent unanswered. Not knowing which important charge transport features to optimize, limits the development of next generations of organic optoelectronic devices. 
Here, we address this by measuring photo-generated charge motion from the first hopping events (with sub-picosecond time resolution) to full extraction in complete solar cell devices based on co-evaporated bulk heterojunctions of $\alpha$-sexithiophene $(\alpha-6 \mathrm{~T})$ and buckminsterfullerene $\left(\mathrm{C}_{60}\right)$. We carefully vary the molar fraction of $\alpha-6 \mathrm{~T}$ in $\mathrm{C}_{60}$ from homogeneously diluted $(<10 \%)$, to a point where $\alpha$-6T begins to form isolated aggregates ( $>10-25 \%$ ) or is strongly aggregated (50\%). We thus vary the distance between isolated $\alpha-6 \mathrm{~T}$ sites and the level of disruption of the $\mathrm{C}_{60}$ phase in a controlled manner - the $\alpha$-6T: $\mathrm{C}_{60}$ system may be viewed as a model for the mixed donor-acceptor phase in OPV. $\mathrm{C}_{60}$ was chosen as the model acceptor since its use in organic electronics is ubiquitous, whereas $\alpha$-6T was picked as the model donor as it consists of a sequence of thiophene units, similar to many conjugated donors, e.g. P3HT. The well-defined sample morphology and the unique time resolution of our transient measurements enables us to quantify the impact of donor-acceptor mixing on the charge transport kinetics.

We experimentally show that even when the donor sites are highly diluted (5.7-10\% molar) and the donor phase is discontinuous, hole transport between isolated donor sites nevertheless occurs by long-range hole tunneling through several buckminsterfullerene molecules (over distances of $\sim 4 \mathrm{~nm}$ ) - an often overlooked hole transport mechanism in organic solar cells. We demonstrate that for conditions relevant to OPV device operation, hole transport between isolated donor sites occurs with a reasonably high hole mobility $\left(\mu_{\mathrm{h}}=5-15 \times 10^{-5} \mathrm{~cm}^{2} \mathrm{~V}^{-1} \mathrm{~s}^{-1}\right.$, depending on the concentration of the donor). We find that at low amounts of the donor $(<10 \%$ molar) electron transport in the $\mathrm{C}_{60}$ phase remains unperturbed $\left(\mu_{\mathrm{e}}=2 \mathrm{~cm}^{2} \mathrm{~V}^{-1} \mathrm{~s}^{-1}\right)$, so the $\mathrm{C}_{60}$ phase may be considered as effectively pure for electron transport. As such, $\mathrm{C}_{60}$ domains containing only a trace amount of donor enable ambipolar transport by long-range hole tunneling. This shows that the general notion that a continuous donor network is strictly necessary for hole transport is incorrect. 
Furthermore, we demonstrate that at high reverse bias and low donor concentration (1.5-25\% molar) a small fraction of the hole population (0-20\% of the total, depending on bias) can be transferred to and extracted via $\mathrm{C}_{60}$ with a high hole mobility $\left(\mu_{\mathrm{h}}=0.1-2 \mathrm{~cm}^{2} \mathrm{~V}^{-1} \mathrm{~s}^{-1}\right.$ for 1.5-25\% donor in $\mathrm{C}_{60}$ ). Nevertheless, for conditions relevant to OPV device operation, facile hole capture by isolated donor sites rapidly reduces the fraction of holes transported in $\mathrm{C}_{60}$ to zero, in which case subsequent hole motion occurs by long-range tunneling between isolated donor sites.

\section{Results}

\section{1. $\alpha-6 \mathrm{~T}: \mathrm{C}_{60}$ as a Model System for the Mixed Phase}

We study co-evaporated bulk heterojunctions of $\alpha$-6T and the neat fullerene $\mathrm{C}_{60}$ as a model system for the mixed donor-acceptor phase in OPV. Controlled evaporation under ultra-high vacuum allows us to precisely vary the molar fraction of $\alpha-6 \mathrm{~T}$ in $\mathrm{C}_{60}$, and thereby tune the distance between isolated $\alpha$-6T (see SI Table S1) and the degree of donor-acceptor mixing (Figure 1a). This is in contrast to solution-cast polymer OPV blends, where the inter-donorsite distance is very challenging, if not impossible, to tune reliably.

It was first shown by Tang et al. ${ }^{[17]}$ and later repeated by others, ${ }^{[18]}$ that even low donor amounts in a $\mathrm{C}_{60}$ matrix (5\% by weight) lead to OPV devices with a peak external quantum efficiency $(\mathrm{EQE}) \approx 70 \%$ for various small molecule donors. Using $\mathrm{C}_{70}$ peak EQE $\approx 75-80 \% .{ }^{[17,19,20]}$ For the $\alpha-6 \mathrm{~T}: \mathrm{C}_{60}$ series we also obtain a peak $\mathrm{EQE}=70 \%$ and $F F=0.55-0.57$ at $\alpha-6 \mathrm{~T}$ content in the range of $4-7 \%$ by weight (5.7-10\% molar) with similar device characteristics as reported by Tang et al., see SI Figure S1 and SI Table S2. As such, the results that are presented here are expected to be general and applicable to other small-molecule-donor and neat fullerene $\left(\mathrm{C}_{60}\right.$ or $\mathrm{C}_{70}$ ) mixtures.

Since $\alpha$-6T has a strong tendency to aggregate, it enables us to accurately identify the transition from diluted and largely un-aggregated $\alpha$-6T to the aggregated $\alpha-6 \mathrm{~T}$ phase by analysis of 
spectroscopic data shown below. The relatively weak absorption coefficient of $\alpha$-6T compared to that of $\mathrm{C}_{60}$ also enables us to simultaneously record spectral blueshifts of the $\mathrm{C}_{60}$ phase $-\mathrm{a}$ measure for the increased disorder of $\mathrm{C}_{60}{ }^{[21]}$

Figure 1b shows EQE measurements that enable us to characterize the morphology of the active layer. The spectra are normalized to the $\mathrm{C}_{60}$ absorption peak at $2 \mathrm{eV}$, corresponding to an intra-molecular singlet absorption of $\mathrm{C}_{60},{ }^{[21]}$ and scaled by the molar fraction of $\mathrm{C}_{60}$. As $\mathrm{C}_{60}$ is the main absorber in these blends, the EQE spectra above $1.8 \mathrm{eV}$ are mainly dominated by the absorption in $\mathrm{C}_{60}$ (optical gap $\mathrm{C}_{60} \approx 1.8 \mathrm{eV}$, optical gap $\alpha-6 \mathrm{~T} \approx 2.3 \mathrm{eV}$ ), whereas below $1.8 \mathrm{eV}$ the spectra are dominated by the absorption of the charge-transfer (CT) state manifold, see SI Figure S2. The CT state absorption strength is proportional to the density of CT states in the blend and is a direct measure of the donor-acceptor interfacial area. ${ }^{[18]}$ When the donor is homogeneously diluted in $\mathrm{C}_{60}$, the interfacial area is, therefore, expected to scale linearly with the donor content. Up to $\alpha-6 \mathrm{~T}$ content of $10 \%$ this is indeed the case, as can be observed when the CT manifold is fitted by a Gaussian distribution according to Marcus theory, ${ }^{[22]}$ see inset of Figure 1b. We observe a deviation from the linear trend at a donor content of $25 \%$ indicative of the onset of $\alpha$-6T aggregation. Donor aggregation is clearly visible only when the donor fraction is $50 \%$, with a strongly redshifted CT absorption.

X-Ray Reflectivity (XRR) measurements at high donor content $(\approx 25-50 \%$ molar) indicate pure $\alpha$-6T aggregates, whereas Grazing-Incidence X-ray Diffraction (GIXD) shows smeared out diffraction peaks, indicating randomly oriented $\alpha-6 \mathrm{~T}$ aggregates with no preferred orientation. ${ }^{[23]}$ Therefore, up to a donor content of $10 \%$ molar, $\alpha-6 \mathrm{~T}$ is homogeneously diluted at random orientations in $\mathrm{C}_{60}$. At higher donor content (10-25\% molar) a mixture of mostly isolated $\alpha-6 \mathrm{~T}$ and some randomly oriented and isolated $\alpha-6 \mathrm{~T}$ aggregates occurs. We will show below that up to $\alpha-6 \mathrm{~T}$ content of $50 \%$, when a large fraction of $\alpha-6 \mathrm{~T}$ aggregates is expected, the donor phase remains discontinuous. As such, we vary the distance between isolated $\alpha-6 \mathrm{~T}$ 
sites and the level of disruption of the $\mathrm{C}_{60}$ phase in a controlled manner, from a purely diluted donor to a discontinuous donor network with aggregates - a model system to study the effects of donor-acceptor mixing on the charge transport kinetics.

\subsection{Time-Resolved Charge Extraction}

To quantify how the purity of the neat phases affects photo-generated charge transport, we have followed their motion from photo-generation to full extraction in complete solar cell devices. We rely on a combination of the Time-Resolved Electric-Field-Induced Second Harmonic generation (TREFISH) technique ${ }^{[24]}$ combined with photocurrent measurements ${ }^{[25]}$, enabling us to follow the motion of photo-generated carriers from the first hopping events, occurring on a sub-picosecond to picosecond time scale after photoexcitation, to full extraction.

Figure 1c shows the measurement scheme. The motion of photo-generated charges partially screens the electric field $E$ induced by the applied bias, resulting in a change $\Delta E(t)$. The resulting change $\Delta E(t)$ is detected optically by a measurement of the second-harmonic intensity $I_{2 \mathrm{H}}(t) \propto \Delta E^{2}(t)$, enabling sub-picosecond temporal resolution. The TREFISH measurement is complemented by a simultaneous (using the same pump pulse) recording of the photocurrent transient using an oscilloscope. As such, all relevant time scales for charge transport, from subpicoseconds to tens of microseconds, are probed in a single measurement.

In our time-resolved measurements we have deliberately chosen low energy pump photons $(1.53 \mathrm{eV})$ to only excite the CT state manifold, as indicated by the black dashed line in Figure $1 \mathrm{~b}$. At $1.53 \mathrm{eV}$ we are predominantly exciting the (isolated $\alpha-6 \mathrm{~T}$ ): $\mathrm{C}_{60}$ interfaces and not those of (aggregated $\alpha-6 \mathrm{~T}): \mathrm{C}_{60}$, as the absorption of the latter occurs at lower photon energies (Figure 1b). SI Figure S2 shows that at $1.53 \mathrm{eV}$ photo-generation in the $\mathrm{C}_{60}$ phase can be neglected for all donor concentrations. This ensures that the starting point of the time-resolved measurement is an excited CT complex at the (isolated $\alpha-6 \mathrm{~T}): \mathrm{C}_{60}$ interface, instantaneously creating an electron in $\mathrm{C}_{60}$ and a hole in isolated $\alpha-6 \mathrm{~T}$. 
Figure 2 shows the time-resolved extraction of photo-generated charges from OPV devices with an increasing molar fraction of $\alpha-6 \mathrm{~T}$ at the indicated applied reverse bias $U$, where $U=-0.1 \mathrm{~V}$ corresponds to (close to) short-circuit conditions ( $U=0 \mathrm{~V}$ was not possible for technical reasons, see the Experimental Section). We have previously experimentally demonstrated that these traces represent the cumulative amount of photo-generated charge collected at the electrodes. ${ }^{[25,26]}$

Before we proceed with the detailed analysis of these kinetics, we highlight that these measurements were performed at relatively low excitation fluences (see the Experimental Section), leading to extracted carrier densities $n$ comparable to those found in typical well-performing OPV devices under steady-state AM1.5 illumination (of the order of $n=10^{16} \mathrm{~cm}^{-3}$, as in Figure 2). ${ }^{[27-29]}$ To confirm that the extraction kinetics of Figure 2 do indeed describe the charge transport physics of operating OPV devices, we first directly compare our transients to steady-state current-voltage (IV) measurements on the same device.

Figure 3a shows good agreement between steady-state photocurrent-voltage measurements under AM1.5 illumination (blue trace, 10\% $\alpha$-6T device, SI Figure S3 shows other devices) and photocurrent- $\mathrm{V}$ curves reconstructed using the transient measurements (solid orange traces). The steady-state photocurrent-V data were obtained by subtracting the steady-state IV measurements in the dark from IVs under illumination. The photocurrent vs V curves from transient measurements were estimated by recording the total amount of collected charge at $t=10 \mu$ s versus applied bias and were scaled to match at $U=-5 \mathrm{~V}$ for comparison. This is allowed because transient measurements were performed in the linear pump-fluence regime, see SI Figure S4. We obtain a similarly good agreement when using only a continuous $785 \mathrm{~nm}$ laser (1.58 eV) (Figure 3a blue symbols), see SI Figure S3 for agreement in other devices. This confirms that the transient data in Figure 2 represents the conditions relevant for steady-state device operation and thus reflects charge extraction/recombination as they occur in operating OPV devices under AM1.5 illumination. 


\subsection{Long-Range Hole Tunneling}

When $\alpha$-6T is homogeneously diluted, it is not evident how the holes photo-generated in the CT state are extracted from the OPV device. We demonstrate below that a small fraction $(0-20 \%$, depending on bias) of photo-generated holes is transferred to and extracted via $\mathrm{C}_{60}$, whereas the remaining majority of the holes (80-100\%) is transported between isolated donor sites by long-range hole tunneling through $\mathrm{C}_{60}$.

Transient data in Figure 2 indicate two extraction plateaus, most clearly visible for the 5.7\% and $10 \%$ devices. Given the approximate temporal position of these extraction plateaus $t=2.3 \times 10^{-11} \mathrm{~s}$ and $t=2 \times 10^{-7} \mathrm{~s}$ (black dashed lines in Figure 2), charge carrier mobilities of $\mu=0.5 \mathrm{~cm}^{2} \mathrm{~V}^{-1} \mathrm{~s}^{-1}$ and $\mu=6 \times 10^{-5} \mathrm{~cm}^{2} \mathrm{~V}^{-1} \mathrm{~s}^{-1}$, respectively, are expected. The latter part of the transients is attributed to hole motion via isolated $\alpha-6 \mathrm{~T}$ sites, as confirmed by steady-state holeonly mobility measurements using space-charge limited currents (SCLC) giving $\mu_{\mathrm{h}}=6 \times 10^{-5} \mathrm{~cm}^{2} \mathrm{~V}^{-1} \mathrm{~s}^{-1}$ (at 10\% molar), see SI Figure S5. On basis of the high electron mobility reported in neat $\mathrm{C}_{60}$ crystals $\left(\mu_{\mathrm{e}}=0.5 \mathrm{~cm}^{2} \mathrm{~V}^{-1} \mathrm{~s}^{-1}\right)$, ${ }^{[30]}$ we attribute the early part of the transients mainly to the extraction of electrons. However, as we will show below, a small fraction of holes (0-20\%, depending on bias) is also extracted very rapidly via $\mathrm{C}_{60}$.

As photo-generated carriers are generated in pairs the amount of extracted holes should be equal to that of electrons. This is clearly visible for $5.7 \%$ and $10 \%$ devices at (close to) short-circuit conditions $(U=-0.1 \mathrm{~V})$, where the extraction plateau ratio is very close to 0.5 (Figure $3 \mathrm{~b}$ ), whereas it is difficult to discern for the remaining samples and is not shown. A ratio of 0.5 means that the early time plateau is entirely dominated by the fast extraction of electrons and that all holes are extracted via isolated donor sites. However, we observe that with increasing reverse bias the fraction of photo-generated charges extracted at early times is increasing above $50 \%$ (Figure 3b), suggesting that an increasing fraction of holes is extracted very rapidly. Given the high hole mobility reported in neat $\mathrm{C}_{60}$ crystals $\left(\mu_{\mathrm{h}}=2 \mathrm{~cm}^{2} \mathrm{~V}^{-1} \mathrm{~s}^{-1}\right)^{[30]}$ this suggests 
that an increasing fraction of holes is extracted via $\mathrm{C}_{60}$, which we will discuss below. Nevertheless, Figure 3b shows that for the fields relevant to OPV devices ( $U=0 \mathrm{~V}$ corresponds to short-circuit conditions), hole trapping reduces the fraction of holes transported in $\mathrm{C}_{60}$ to zero, forcing all holes to move via isolated $\alpha-6 \mathrm{~T}$.

The mechanism for the initial hole transfer to $\mathrm{C}_{60}$ at high reverse bias is not entirely clear, as following photoexcitation in the CT manifold, the hole is expected to initially reside in $\alpha-6 \mathrm{~T}$. Furthermore, based on the difference between the HOMO levels of $\alpha-6 \mathrm{~T}(-5.3 \mathrm{eV})$ and $\mathrm{C}_{60}$ (-6.2 eV), as determined by Ultraviolet Photoelectron Spectroscopy (UPS), ${ }^{[31]}$ the energetic barrier for hole transfer from $\alpha-6 \mathrm{~T}$ to $\mathrm{C}_{60}$ is expected to be roughly $0.9 \mathrm{eV}=35 \mathrm{kT}$. Hence, thermal de-trapping of the hole seems unlikely.

Instead, we propose that hole transfer to $\mathrm{C}_{60}$ may be assisted by Fowler-Nordheim-type tunneling through a triangular barrier (Figure 1a), as confirmed by good fits to the FowlerNordheim equation, ${ }^{[32]}$ describing the tunneling probability (green trace in Figure 3b). For the equally good fits at other donor fractions see SI Figure S6. In principle, the tunneling probability of a particle with excess energy (such as after photo-excitation) is higher than that of a particle tunneling from the lowest energy of the trap site (such as after a prolonged trapping event). We speculate that photo-induced hole transfer may occur before on-site thermalization, faster than $\sim 1 \mathrm{ps},{ }^{[33]}$ effectively lowering the tunneling barrier (compare the two situations at high field $F$ in Figure 1a). This process divides the extracted hole population into two distinct parts: holes transferred to $\mathrm{C}_{60}$ and extracted very rapidly and holes trapped in isolated $\alpha$-6T and extracted slowly. For a more detailed discussion, see SI Figures S6 and S7, and SI Notes S1 and S2. Nevertheless, for the practically relevant fields to OPV devices all holes remain trapped in isolated $\alpha-6 \mathrm{~T}$.

Following spatial trapping in isolated $\alpha$-6T sites, there are two possible mechanisms for further hole transport. Either the hole escapes $\alpha$-6T and is transported via $\mathrm{C}_{60}$ until a further trapping 
event, or the hole instead tunnels through $\mathrm{C}_{60}$ to another $\alpha$-6T. On basis of the large energy barrier for thermal hole de-trapping from $\alpha-6 \mathrm{~T}$ to $\mathrm{C}_{60}(0.9 \mathrm{eV}=35 \mathrm{kT})$ and the unlikelihood of Fowler-Nordheim-type tunneling at low fields between short-circuit and $V_{\text {OC }}$, both de-trapping scenarios seem unlikely. At low fields long-range hole tunneling through several $\mathrm{C}_{60}$ molecules to neighboring trap sites seems more plausible. Note that in this case the tunneling mechanism is different as at low fields the barrier is expected to be rectangular (Figure 1a) with a tunneling probability that is independent of field, see SI Figure S6 and SI Note S2. A similar transition from direct tunneling to field emission at moderate bias $(<1 \mathrm{~V})$ has been demonstrated by Kushmerick et al. in molecular junctions of $\pi$-conjugated thiols. ${ }^{[34]}$

The mean center-to-center distance between isolated $\alpha$-6T decreases from roughly $\approx 5.3 \mathrm{~nm}$ to $\approx 3.4 \mathrm{~nm}$ when going from $1.5 \%$ to $5.7 \%$ molar $\alpha$-6T in $\mathrm{C}_{60}$. SI Table S1 shows details on the conversion between weight, molar and volume fractions, and the deduced distance between isolated $\alpha$-6T molecules. The extraction kinetics in Figure 2 clearly indicate that upon going from $1.5 \%$ to $5.7 \%$ molar $\alpha$-6T, the second extraction plateau (at microsecond time scales) grows and shifts to significantly shorter times. This suggests that the onset for efficient long-range hole tunneling through $\mathrm{C}_{60}$ to neighboring $\alpha$-6T lies roughly in the 3.4-5.3 nm range, which corresponds to a distance of several $\mathrm{C}_{60}$ molecules $\left(\mathrm{C}_{60}\right.$ diameter is $0.71 \mathrm{~nm}$, center-tocenter distance is $\approx 1 \mathrm{~nm}$ ). A similar onset from direct to thermally activated tunneling $\approx 4 \mathrm{~nm}$ has been reported by Frisbie et al. in $\pi$-conjugated molecular wires. ${ }^{[35]}$ Although at low donor fractions (5.7-10\% molar) long-range hole tunneling is already evident from the experimental data alone, at higher donor content (25-50\% molar) the situation is less clear; not only the packing of $\mathrm{C}_{60}$ may be significantly distorted, but also the $\alpha$-6T phase may form a discontinuous network, reducing the fraction of holes that undergo long-range tunneling. At this point an advanced charge transport model is required to quantitatively explain the kinetics. 


\subsection{Simulations Using the Gaussian Disorder Model}

To simulate the extraction of three charge carrier populations - electrons in $\mathrm{C}_{60}$, holes in $\mathrm{C}_{60}$ and holes tunneling through $\mathrm{C}_{60}$ between isolated donor sites - we rely on the extended Gaussian Disorder Model (eGDM), ${ }^{[36]}$ which has been successfully utilized to explain carrier hopping in a large variety of organic semiconductors. Note that although hole tunneling discussed here occurs over larger distances and through $\mathrm{C}_{60}$ (as opposed to a barrier of empty space), it can still be described by the same eGDM formalism, see SI Note S3.

In brief, the model ${ }^{[37]}$ takes into account: electron-hole recombination; all Coulomb interactions; charge transport via a field- and density-dependent carrier mobility, consistent with the parametrization by Pasveer; ${ }^{[38]}$ charge extraction and injection at contacts. The field-dependence of charge extraction observed in Figure 2 is fitted globally (by a single parameter set), using an iterative least-squares procedure, thus eliminating possible errors due to manual fitting and severely constraining the fit parameters. We have previously shown that our model can successfully fit both transient TREFISH and steady-state SCLC experiments using the same parameter set, ${ }^{[37]}$ which was also done here, as described in SI Figure S5 and SI Table S3.

Figure 2 shows that our simulations capture the charge extraction dynamics observed in experiment reasonably well. The applied bias $U$ in simulations was corrected for the built-in field $U_{\mathrm{bi}}$ of the OPV device, experimentally determined as the bias at which the extracted charge density in the transient measurement is equal to zero $\left(U_{\mathrm{bi}}=1 \mathrm{~V}\right.$ in Figure 3a). Model fits allow us to more accurately quantify the fraction of photo-generated holes transported in $\mathrm{C}_{60}$ (blue traces in Figure 3b), especially for the 1.5\%, 25\% and 50\% devices, for which the extraction plateaus are not clearly visible in experiment (Figure 2). In agreement with the earlier observation from transient data, the hole fraction extracted via $\mathrm{C}_{60}$ is increasing with reverse bias. 
From experimental data alone we cannot distinguish whether the small fraction of holes transferred to $\mathrm{C}_{60}$ (Figure $3 \mathrm{~b}$ ) is extracted without a single trapping event or whether multiple trapping/de-trapping events occur during hole extraction via $\mathrm{C}_{60}$. To quantify, we have extended our modeling to account for the morphology of the BHJ active layer: a fraction of the total simulated volume 90x90x50 (nm³) was occupied by randomly dispersed $\alpha$-6T sites, using the known volume fractions. These simulations (SI Figure S7) suggest that the fast fraction of holes $(0-20 \%$ of the total in Figure $3 b)$ consist of holes extracted via $\mathrm{C}_{60}$ without a single trapping event $(0-10 \%$ of the total) and holes captured by $\alpha-6 \mathrm{~T}$ but undergoing ultra-fast Fowler-Nordheim-type de-trapping back to $\mathrm{C}_{60}$ (possible only at high fields $F$ ). Hence, the majority of the holes (80-100\%) undergo trapping in $\alpha$-6T but cannot be re-transferred to $\mathrm{C}_{60}$ even at high fields and require long-range tunneling to neighboring donor sites. This slows down hole transport by 4-5 orders of magnitude in time, leading to a significantly reduced quasiequilibrium hole mobility from $\mu_{\mathrm{h}}=0.4 \mathrm{~cm}^{2} \mathrm{~V}^{-1} \mathrm{~s}^{-1}$ in the $\mathrm{C}_{60}$ phase to $\mu_{\mathrm{h}}=6 \times 10^{-5} \mathrm{~cm}^{2} \mathrm{~V}^{-1} \mathrm{~s}^{-1}$ for long-range tunneling (numbers at $10 \%$ dilution).

\subsection{Relation between Phase Purity and Charge Motion}

Figure 4a shows how the addition of $\alpha$-6T disrupts the $\mathrm{C}_{60}$ phase. The increase in the electron energetic disorder of $\mathrm{C}_{60}$ has a clear onset at a donor fraction of $10 \%$ molar, in agreement with the transient data, where the temporal position of the electron extraction peak at the relevant (low) fields shifts to longer times at 25\% molar (black dashed vertical line in Figure 2 is a guide to the eye). The increase in the energetic disorder is also reflected in the spectral blueshift of the EQE spectra at $2.5 \mathrm{eV}$, corresponding to an inter-molecular ${ }^{[21]}$ absorption peak of $\mathrm{C}_{60}$ (SI Figure S8). GIXD data shows that at $\approx 25-50 \%$ molar the diffraction rings corresponding to poly-crystalline $\mathrm{C}_{60}$ are no longer observed ${ }^{[23]}$ - indicating amorphous $\mathrm{C}_{60}$, for which the electron energetic disorder seems to saturate at $\sigma \approx 95 \mathrm{meV}$ (Figure 4a). 
Figure 4b shows how donor-acceptor mixing affects the photo-generated electron mobility. The addition of the donor below $10 \%$ molar does not hinder electron transport in the $\mathrm{C}_{60}$ phase significantly - a quasi-equilibrium (long time) electron mobility of the order of $\mu_{\mathrm{e}}=1-2 \mathrm{~cm}^{2} \mathrm{~V}^{-1} \mathrm{~s}^{-1}$ is retained, similar to that reported in neat $\mathrm{C}_{60}$ crystals. ${ }^{[30]}$ In contrast, at donor fractions higher than $10 \%$, electron transport becomes increasingly dispersive with a time-dependent mobility due to carrier thermalization in the disorder-broadened density of states (DOS). ${ }^{[39]}$ The quasi-equilibrium electron mobility is then roughly an order of magnitude lower $\mu_{\mathrm{e}}=0.08 \mathrm{~cm}^{2} \mathrm{~V}^{-1} \mathrm{~s}^{-1}$ (at 25\% molar) than at low donor content. Thus, at donor content below $10 \%$ molar the $\mathrm{C}_{60}$ phase can be considered as effectively pure for electron transport. The hole energetic disorder in $\alpha$-6T remains relatively constant around the mean $\sigma_{\mathrm{h}}=110 \mathrm{meV}$, except for the $1.5 \%$ case. This is because hole transport at $5.7-50 \%$ donor content is governed by hole trapping in isolated $\alpha$-6T, followed by long-range tunneling through $\mathrm{C}_{60}$ to neighboring donor sites, whereas at $1.5 \%$ long-range tunneling is hindered. Thus, except for the $1.5 \%$ case, the resulting hole mobility kinetics are very similar and show only a slight increase with increasing donor content (Figure 4b), as corroborated by the lack of a significant temporal shift of the hole extraction peak in the transient extraction kinetics (black dashed vertical line in Figure 2). In this case the time-dependent hole mobility quantitatively describes the gradual relaxation of the hole population into low-lying $\alpha$-6T sites.

The observed trends in the transient hole mobility and hole energetic disorder of $\alpha$-6T are further experimentally confirmed by steady-state SCLC hole-only mobility measurements. The inset of Figure $4 \mathrm{~b}$ shows that hole mobility $\mu_{0}$ (in the low-concentration regime, see the Experimental Section) increases substantially at donor content higher than $1.5 \%$, which marks the onset for efficient long-range hole tunneling. Following this onset hole mobility effectively plateaus - increases only slightly as the tunneling distance decreases with increasing donor content $\left(\mu_{\mathrm{h}}=5-15 \times 10^{-5} \mathrm{~cm}^{2} \mathrm{~V}^{-1} \mathrm{~s}^{-1}\right.$ at $5.7-50 \%$ molar$)$. 
Since at low donor content we vary the distance $r$ between isolated $\alpha$-6T sites, the inset of Figure $4 \mathrm{~b}$ allows us to estimate the hole localization length $\alpha^{-1}$ in the framework of eGDM as $\mu \propto r^{2} \exp (-2 \alpha r)$. We obtain a surprisingly large number $\alpha^{-1}=1 \pm 0.1 \mathrm{~nm}$ (see SI Figure S9), whereas typically $\alpha^{-1} \sim 0.1 \mathrm{~nm}$ is assumed, ${ }^{[36,38]}$ based on earlier data for TNF:PVK $\left(\alpha^{-1} \approx 0.11 \mathrm{~nm}\right)$ by Gill ${ }^{[40]}$ and for P3HT and $\mathrm{OC}_{1} \mathrm{C}_{10}$-PPV $\left(\alpha^{-1} \approx 0.15 \mathrm{~nm}\right)$ by de Leeuw et al. in ref. [41]. Full material names are given in the Experimental Section. For large $\alpha^{-1}$ the hole wavefunction extends a larger distance from the donor site, enabling long-range tunneling. We also observe an approximately 40-fold increase in $\mu_{0}$ when the donor concentration increases to $75 \%$, possibly indicating the formation of a continuous donor network, eliminating the need for long-range tunneling. Although we were unable to perform transient measurements on samples with high donor loading due to their weak $2^{\text {nd }}$ harmonic intensity, the SCLC data for the $75 \%$ donor case do indicate a considerable $(\sim 43 \times)$ increase in the hole attempt-to-hop frequency $v_{\mathrm{h}}$ and a similar hole disorder as for the other donor fractions $\sigma_{\mathrm{h}} \approx 105 \mathrm{meV}$, see SI Table S3. The higher attempt-to-hop frequency reflects a shorter hopping distance due to the formation of a continuous donor network (SI Note S3), increasing the carrier mobility up to $\mu_{0}=4 \times 10^{-3} \mathrm{~cm}^{2} \mathrm{~V}^{-1} \mathrm{~s}^{-1}$. Field-effect mobilities of approximately $10^{-2} \mathrm{~cm}^{2} \mathrm{~V}^{-1} \mathrm{~s}^{-1}$ were reported for poly-crystalline $\alpha-6 \mathrm{~T} .{ }^{[42]}$ The hole-only SCLC data thus indicates a change in the dominant hole transport mechanism - from long-range hole tunneling at 50\% donor to hole transport via a continuous donor network at $75 \%$ donor. Most importantly, the combined dataset clearly indicates that $\mathrm{C}_{60}$ domains containing an intermediate donor concentration (5.7-50\% molar, corresponding to isolated donor sites and a discontinuous donor network with aggregates) enable reasonable hole transport $\left(\mu_{\mathrm{h}}=5-15 \times 10^{-5} \mathrm{~cm}^{2} \mathrm{~V}^{-1} \mathrm{~s}^{-1}\right.$ at $5.7-50 \%$ molar).

Since the results presented here are expected to be general, we propose that Figure 4 may be used as a reference to what occurs in the fullerene phase upon the presence of a small amount of material/molecule with a donating character. As the photo-generated carriers in BHJs are 
expected to traverse both neat and mixed donor-acceptor domains, a combination of the above kinetics would be representative of the full charge transport kinetics.

\section{Discussion}

To the best of our knowledge, our data is the first experimental demonstration of following the transient motion of charges photo-generated in the CT manifold in a complete OPV device. In agreement with earlier reports using Internal Quantum Efficiency (IQE) measurements, ${ }^{[43]}$ we also observe that such photo-generated charges are extracted efficiently.

Generally, excitation in the CT manifold is expected to produce free carriers in the lowest lying DOS states, with little to no 'excess energy' for further thermalization, and thus a low transient mobility. Our results show that this is not the case - if the material is sufficiently disordered, even charges photo-generated in the CT manifold undergo further thermalization with a time-dependent mobility. This is clearly visible in Figure 4, where $\sigma$ gradually increases when going from almost non-disordered $\mathrm{C}_{60}$, then to disordered $\mathrm{C}_{60}$ and then to the highly disordered $\alpha$-6T, leading to an increasingly time-dependent carrier mobility. The associated increasingly dispersive nature of charge transport is also directly visible in the increasingly convex shape of the extraction plateaus in Figure 2.

The possible importance of ambipolar transport in the fullerene phase of organic BHJ solar cells has been previously highlighted by several groups, ${ }^{[13,44,45]}$ challenging the general notion that hole transport is strictly facilitated only by the donor, and electron transport only by the acceptor. In fact, high hole mobility in neat $\mathrm{C}_{60}$ has been well known since the $1990 \mathrm{~s} .{ }^{[30]}$ Our results show that although $\mathrm{C}_{60}$ enables efficient hole transport, the fraction of holes extracted via $\mathrm{C}_{60}$ at relevant fields for OPV devices is effectively zero. This is due to facile hole capture by the donor during hole transport in $\mathrm{C}_{60}$. Therefore, at least for the case of small-molecule-donor and neat fullerene ( $\mathrm{C}_{60}$ or $\mathrm{C}_{70}$ ) OPV devices, hole extraction only via the fullerene phase may be ignored. 
Our results highlight the significance of another charge transport mechanism - long-range hole tunneling through several $\mathrm{C}_{60}$ molecules. The effect of long-range charge tunneling between isolated small-molecule sites is similar to that of tie-chains in conjugated polymers, which were suggested to be responsible for charge transport between domains of ordered polymer. ${ }^{[46]}$ Both long-range tunneling and tie-chains effectively act as bridges between 'favorable' sites and may occur concurrently. We expect that the tunneling processes discussed here are also relevant to OPVs using polymeric donors and/or acceptors, but the respective concentrations that are needed in a mixed phase to enable ambipolar transport may be different from the present system. The emissive layer in some state-of-the art organic light-emitting diodes (OLEDs) also consists of a mixture of a small concentration (3-10\% molar) of guest molecules, typically a phosphorescent dye, embedded in a host matrix. Using ab initio modeling, Wenzel et al. have recently demonstrated that charge transport between distant sites in such host-guest systems is mediated via the coherent process of molecular superexchange. ${ }^{[47]}$ Possibly the long-range hole tunneling that we experimentally demonstrate here for OPVs may be also explained by the theoretical framework laid out in ref. [47], as suggested by its authors.

Our results not only show that a continuous interpenetrating donor-acceptor network is not strictly necessary but also redefine the meaning of the commonly used terms of 'neat', 'pristine' or 'pure phase'. More concretely, we have shown that $\mathrm{C}_{60}$ may be considered as effectively pure for electron transport only if the molar fraction of the donor does not exceed $10 \%$. From a hole transport perspective, the same material, containing $90 \%$ of $\mathrm{C}_{60}$ in $\alpha$-6T, would generally be regarded as 'not pure at all'. However, even at such low amounts of isolated donor, the hole mobility may be as high as $\mu_{\mathrm{h}} \approx 6 \times 10^{-5} \mathrm{~cm}^{2} \mathrm{~V}^{-1} \mathrm{~s}^{-1}$ (at $10 \%$ molar) and is increasing with donor content ( $\mu_{\mathrm{h}} \approx 1.1 \times 10^{-4} \mathrm{~cm}^{2} \mathrm{~V}^{-1} \mathrm{~s}^{-1}$ at $25 \%$ molar), which is comparable to that reported for some neat donor materials used in OPV devices in the past. Therefore, the common notion that a continuous donor network is strictly necessary for efficient OPV device operation is erroneous. 


\section{Conclusion}

We have shown, under conditions relevant to OPV device operation, that even when the donor sites are highly diluted (5.7-10\% molar) and the donor phase is discontinuous, hole transport between isolated donor sites can nevertheless occur by long-range hole tunneling through several buckminsterfullerene molecules (over distances as large as $4 \mathrm{~nm}$ ). Hole transport between isolated donor sites occurs with a reasonably high hole mobility ( $\mu_{\mathrm{h}}=5-15 \times 10^{-5} \mathrm{~cm}^{2} \mathrm{~V}^{-1} \mathrm{~s}^{-1}$, depending on the concentration of the donor). At low donor amounts $(<10 \%$ molar $)$ electron transport in the $\mathrm{C}_{60}$ phase remains unperturbed $\left(\mu_{\mathrm{e}}=2 \mathrm{~cm}^{2} \mathrm{~V}^{-1} \mathrm{~s}^{-1}\right.$ ) and the $\mathrm{C}_{60}$ phase may be considered as effectively pure for electron transport. As such, $\mathrm{C}_{60}$ domains containing only a trace amount of donor enable ambipolar transport by long-range hole tunneling - a continuous donor network is not strictly necessary for hole transport in organic solar cells.

Furthermore, we have shown that at high reverse bias and low donor concentration (1.5-25\% molar) a small fraction of the hole population (0-20\% of the total, depending on bias) can be transferred to and extracted via $\mathrm{C}_{60}$ with a high hole mobility $\left(\mu_{\mathrm{h}}=0.1-2 \mathrm{~cm}^{2} \mathrm{~V}^{-1} \mathrm{~s}^{-1}\right.$ for 1.5-25\% donor in $\mathrm{C}_{60}$ ). Nevertheless, at field strengths relevant to OPV devices, facile hole capture by isolated donor sites rapidly reduces the fraction of holes transported in $\mathrm{C}_{60}$ to zero. Subsequent hole motion occurs by long-range tunneling between isolated donor sites.

Since hole transport can occur via the acceptor phase, containing just a small fraction of material with a donating character, these results question the relevance of the commonly used terms of 'neat', 'pristine' or 'pure phase' and whether a continuous interpenetrating donor-acceptor network is the ideal morphology of charge transport. The limits of long-range hole tunneling are yet to be explored. 


\section{Experimental Section}

Full material names. Trinitrofluorenone (TNF), poly- $n$-vinylcarbazole (PVK), Poly(3-hexylthiophene) (P3HT), poly(2-methoxy-5-(3',7’-dimethyloctyloxy)-p-phenylene vinylene) $\left(\mathrm{OC}_{1} \mathrm{C}_{10}-\mathrm{PPV}\right)$.

Device fabrication. The photovoltaic devices are thermally evaporated at ultra-high vacuum (base pressure $<10^{-7}$ mbar) onto a glass substrate with a pre-structured ITO contact (Thin Film Devices, USA). $2 \mathrm{~nm}$ of $\mathrm{MoO}_{3}$ are deposited to adjust the ITO work function and form an Ohmic hole contact. The active layer comprises $50 \mathrm{~nm}$ of $\mathrm{C}_{60}$ (CreaPhys $\mathrm{GmbH}$, Germany) blended with $\alpha$-6T (Lumtec, TW) at $\alpha$-6T molar fraction ranging from $1.5 \%$ to $75 \%$ (see SI Table S1). Afterwards, $8 \mathrm{~nm}$ of Bathophenanthroline (BPhen, abcr GmbH, Germany), used as electron contact, is evaporated and finished with $100 \mathrm{~nm}$ of Al. The device is defined by the geometrical overlap of the bottom and the top contact with an active area of $1.68 \mathrm{~mm}^{2}$. To avoid exposure to ambient conditions, the organic part of the device is covered by a small glass substrate, glued on top, providing encapsulation.

EQE measurements. Measurements were performed using a xenon lamp (Oriel Xe Arch-lamp Apex, Newport, USA), a monochromator (Cornerstone 260 1/4m, Newport, USA), an optical chopper, and a lock-in amplifier (SR 7265, DPS Signal Recovery, USA). A silicon photodiode (Hamamatsu S1337, JP) is used as reference. This technique is used for the absolute determination of the EQE values.

Sensitive EQE measurements. Monochromatic light with varying wavelengths is produced by illuminating a Newport Cornerstone 260 1/4m monochromator with chopped (140 Hz) white light of a quartz halogen lamp $(50 \mathrm{~W})$. The monochromatic light beam is focused onto the organic solar cell and its short-circuit current is amplified before it is analyzed with a lock-in amplifier (Signal Recovery 7280 DSP, Signal Recovery, Oak Ridge, USA). The time constant of the lock-in amplifier was chosen to be $1 \mathrm{~s}$ and the amplification of the pre-amplifier was increased to resolve the low photocurrents at low photon energies. The EQE spectrum is 
obtained by dividing the photocurrent of the solar cell by the flux of incoming photons, which was obtained with a calibrated silicon (Si) and indium-gallium-arsenide (InGaAs) photodiode. Time-resolved measurements. Detailed description of the combined TREFISH and photocurrent experiment can be found in refs [24,25]. Samples were pumped with $810 \mathrm{~nm}$ photons. Pump-fluences in the range of $25.4-87.4 \mu \mathrm{J} \mathrm{cm}^{-2}$ per pulse $\left(1-3.56 \times 10^{13}\right.$ photons $\mathrm{cm}^{-}$ ${ }^{2}$ per pulse) were used, whereas for the $1.5 \%$ device a higher pump-fluence of $381 \mu \mathrm{J} \mathrm{cm}{ }^{-2}$ per pulse $\left(15.5 \times 10^{13}\right.$ photons $\mathrm{cm}^{-2}$ per pulse) was necessary as the CT manifold of the $1.5 \%$ sample is rather weakly absorbing. Note that since CT absorption is orders of magnitude weaker than absorption of the constituent materials, the pump-fluences used in this study may be considered as relatively low. For the TREFISH experiment we have used $810 \mathrm{~nm}$ probe photons. To obtain a reasonably good signal-to-noise ratio in the TREFISH experiment the probe-fluence was in the range of 299-314 $\mu \mathrm{J} \mathrm{cm}^{-2}$ per pulse (12.2-12.8 $\times 10^{13}$ photons $\mathrm{cm}^{-2}$ per pulse), whereas for the $1.5 \%$ device a higher probe-fluence of $564 \mu \mathrm{J} \mathrm{cm}^{-2}$ per pulse $\left(23 \times 10^{13}\right.$ photons $\mathrm{cm}^{-2}$ per pulse) was used. Due to limitations of our mechanical delay stage (3 ns max), the signal-to-noise ratio in the TREFISH experiment at longer time delays (>0.5-1 ns for the present devices) and RC limitations of electrical extraction ( $<20 \mathrm{~ns})$ there is a lack of reliable data roughly in this range 1-20 ns. In cases where we consider the photocurrent measurement reliable below $20 \mathrm{~ns}$, it is also shown, but is marked by thinner traces. Measurements at $U=0 \mathrm{~V}$ were not possible as the output resistance of our function generator (Tektronix AFG 3101) was found to change significantly. $U=-0.1 \mathrm{~V}$ was used instead to ensure reliable measurements at (close to) short-circuit conditions.

Steady-state IV measurements. IV characteristics are measured with a SMU (Keithley 2400, USA) at standard testing conditions (16 S-150 V.3 Solar Light Co., USA) with a mismatch corrected light intensity. For IV measurements at 785 nm illumination a continuous $1 \mathrm{~mW}$ laser was used. 
Simulations. The most comprehensive description of the model can be found in ref. [37]. We have used the Miller-Abrahams formalism to quantify with the least-number of unknown parameters the hopping rate of a charge carrier in a disorder-broadened Gaussian DOS. The real thickness of the BHJ layer was used in the simulations. We simulate three charge carrier populations that can interact fully but are otherwise described by their own carrier hopping parameters: the Gaussian energetic disorder $\sigma$ and the attempt-to-hop frequency $v$, which depends on the inter-site distance, see SI Note S3. For each population the BHJ active layer is thus treated as an effective medium, meaning that local variations in the physical properties of the nanoscale morphology are not explicitly accounted for-obtained carrier hopping parameters represent 'average' values over the entire BHJ active layer. This minimizes the number of unknown simulation parameters to only those that are necessary to explain the experiments. A custom-made code was used to iteratively fit the experiments by least-squares. The most important simulation parameters used/obtained from the model fits to transient and steady-state SCLC experiments are described in the SI Table S3. All steady-state and transient mobilities were calculated for a fractional DOS occupancy of $\mathrm{c}_{0}=10^{-4}$ at $300 \mathrm{~K}$ and an electric field strength of $0.5 \mathrm{~V}$ per $50 \mathrm{~nm}\left(1 \times 10^{5} \mathrm{~V} \mathrm{~cm}^{-1}\right)$.

\section{Supporting Information}

Supporting Information is available from the the author.

\section{Acknowledgements}

D.S, J.B and K.V. were supported by the German Federal Ministry for Education and Research (BMBF) through the InnoProfille project “Organische p-i-n Bauelemente 2.2”. The work in Vilnius was supported by the Research Council of Lithuania (project MIP-85/2015). A.M was supported by the Science Council of Sweden, and O.I thanks the Knut and Alice Wallenberg foundation for instrumental funding and a Wallenberg Scholar grant. 


\section{References}

[1] W. Zhao, D. Qian, S. Zhang, S. Li, O. Inganäs, F. Gao, J. Hou, Adv. Mater. 2016, 28, 4734.

[2] J. Zhao, Y. Li, G. Yang, K. Jiang, H. Lin, H. Ade, W. Ma, H. Yan, Nat. Energy 2016, 1, 15027.

[3] Y. Liu, J. Zhao, Z. Li, C. Mu, W. Ma, H. Hu, K. Jiang, H. Lin, H. Ade, H. Yan, Nat. Commun. 2014, 5, 5293.

[4] F. C. Jamieson, E. B. Domingo, T. McCarthy-Ward, M. Heeney, N. Stingelin, J. R. Durrant, Chem. Sci. 2012, 3, 485.

[5] S. Sweetnam, K. R. Graham, G. O. Ngongang Ndjawa, T. Heumüller, J. A. Bartelt, T. M. Burke, W. Li, W. You, A. Amassian, M. D. McGehee, J. Am. Chem. Soc. 2014, 136, 14078.

[6] J. K. Gallaher, S. K. K. Prasad, M. A. Uddin, T. Kim, J. Y. Kim, H. Y. Woo, J. M. Hodgkiss, Energy Environ. Sci. 2015, 8, 2713.

[7] A. C. Jakowetz, M. L. Böhm, A. Sadhanala, S. Huettner, A. Rao, R. H. Friend, Nat. Mater. 2017, DOI 10.1038/nmat4865.

[8] J. A. Bartelt, Z. M. Beiley, E. T. Hoke, W. R. Mateker, J. D. Douglas, B. A. Collins, J. R. Tumbleston, K. R. Graham, A. Amassian, H. Ade, J. M. J. Fréchet, M. F. Toney, M. D. McGehee, Adv. Energy Mater. 2013, 3, 364.

[9] S. Mukherjee, C. M. Proctor, G. C. Bazan, T.-Q. Nguyen, H. Ade, Adv. Energy Mater. 2015, DOI 10.1002/aenm.201500877.

[10] S. Mukherjee, X. Jiao, H. Ade, Adv. Energy Mater. 2016, DOI 10.1002/aenm.201600699.

[11] C. M. Proctor, A. S. Kher, J. A. Love, Y. Huang, A. Sharenko, G. C. Bazan, T.-Q. Nguyen, Adv. Energy Mater. 2016, DOI 10.1002/aenm.201502285.

[12] S. Foster, F. Deledalle, A. Mitani, T. Kimura, K.-B. Kim, T. Okachi, T. Kirchartz, J. Oguma, K. Miyake, J. R. Durrant, S. Doi, J. Nelson, Adv. Energy Mater. 2014, DOI 10.1002/aenm.201400311.

[13] X. Guo, M. Zhang, J. Tan, S. Zhang, L. Huo, W. Hu, Y. Li, J. Hou, Adv. Mater. 2012, 24, 6536.

[14] J. Huang, G. Li, Y. Yang, Appl. Phys. Lett. 2005, 87, 112105.

[15] V. Pranculis, Y. Infahsaeng, Z. Tang, A. Devižis, D. A. Vithanage, C. S. Ponseca, O. Inganäs, A. P. Yartsev, V. Gulbinas, V. Sundström, J. Am. Chem. Soc. 2014, 136, 11331.

[16] V. Abramavičius, D. A. Vithanage, A. Devižis, Y. Infahsaeng, A. Bruno, S. Foster, P. E. Keivanidis, D. Abramavičius, J. Nelson, A. Yartsev, V. Sundström, V. Gulbinas, Phys. Chem. Chem. Phys. 2014, 16, 2686.

[17] M. Zhang, H. Wang, H. Tian, Y. Geng, C. W. Tang, Adv. Mater. 2011, 23, 4960.

[18] K. Vandewal, J. Widmer, T. Heumueller, C. J. Brabec, M. D. McGehee, K. Leo, M. Riede, A. Salleo, Adv. Mater. 2014, 26, 3839.

[19] Y. Zheng, W. J. Potscavage Jr., T. Komino, M. Hirade, J. Adachi, C. Adachi, Appl. Phys. Lett. 2013, 102, 143304.

[20] Y. Zheng, W. J. Potscavage Jr., T. Komino, C. Adachi, Appl. Phys. Lett. 2013, 102, 153302.

[21] S. Kazaoui, R. Ross, N. Minami, Phys. Rev. B 1995, 52, R11665.

[22] K. Vandewal, K. Tvingstedt, A. Gadisa, O. Inganäs, J. V. Manca, Phys. Rev. B 2010, 81, 125204.

[23] C. Lorch, K. Broch, V. Belova, G. Duva, A. Hinderhofer, A. Gerlach, M. Jankowski, F. Schreiber, J. Appl. Crystallogr. 2016, 49, 1266.

[24] A. Devižis, A. Serbenta, K. Meerholz, D. Hertel, V. Gulbinas, Phys. Rev. Lett. 2009, 103, 027404. 
[25] A. Melianas, V. Pranculis, A. Devižis, V. Gulbinas, O. Inganäs, M. Kemerink, Adv. Funct. Mater. 2014, 24, 4507.

[26] A. Melianas, V. Pranculis, Y. Xia, N. Felekidis, O. Inganäs, V. Gulbinas, M. Kemerink, Adv. Energy Mater. 2017, DOI 10.1002/aenm.201602143.

[27] J. Kniepert, I. Lange, N. J. van der Kaap, L. J. A. Koster, D. Neher, Adv. Energy Mater. 2014, 4, 1301401.

[28] A. Foertig, J. Kniepert, M. Gluecker, T. Brenner, V. Dyakonov, D. Neher, C. Deibel, Adv. Funct. Mater. 2014, 24, 1306.

[29] S. Albrecht, J. R. Tumbleston, S. Janietz, I. Dumsch, S. Allard, U. Scherf, H. Ade, D. Neher, J. Phys. Chem. Lett. 2014, 5, 1131.

[30] E. Frankevich, Y. Maruyama, H. Ogata, Chem. Phys. Lett. 1993, 214, 39.

[31] J. Sakai, T. Taima, K. Saito, Org. Electron. 2008, 9, 582.

[32] R. H. Fowler, L. Nordheim, Proc. R. Soc. Lond. Math. Phys. Eng. Sci. 1928, 119, 173.

[33] P. A. Lane, P. D. Cunningham, J. S. Melinger, O. Esenturk, E. J. Heilweil, Nat. Commun. 2015, 6, 7558.

[34] J. M. Beebe, B. Kim, J. W. Gadzuk, C. Daniel Frisbie, J. G. Kushmerick, Phys. Rev. Lett. 2006, 97, 026801.

[35] S. H. Choi, C. Risko, M. C. R. Delgado, B. Kim, J.-L. Brédas, C. D. Frisbie, J. Am. Chem. Soc. 2010, 132, 4358.

[36] R. Coehoorn, W. F. Pasveer, P. A. Bobbert, M. A. J. Michels, Phys. Rev. B 2005, 72, 155206.

[37] N. Felekidis, A. Melianas, M. Kemerink, Phys. Rev. B 2016, 94, 035205.

[38] W. F. Pasveer, J. Cottaar, C. Tanase, R. Coehoorn, P. A. Bobbert, P. W. M. Blom, D. M. de Leeuw, M. A. J. Michels, Phys. Rev. Lett. 2005, 94, 206601.

[39] A. Melianas, F. Etzold, T. J. Savenije, F. Laquai, O. Inganäs, M. Kemerink, Nat. Commun. 2015, 6, 8778.

[40] W. D. Gill, J. Appl. Phys. 1972, 43, 5033.

[41] C. Tanase, E. J. Meijer, P. W. M. Blom, D. M. de Leeuw, Phys. Rev. Lett. 2003, 91, 216601.

[42] G. Horowitz, E. H. Mohsen, H. Riadh, J. Appl. Phys. 2000, 87, 4456.

[43] K. Vandewal, S. Albrecht, E. T. Hoke, K. R. Graham, J. Widmer, J. D. Douglas, M. Schubert, W. R. Mateker, J. T. Bloking, G. F. Burkhard, A. Sellinger, J. M. J. Fréchet, A. Amassian, M. K. Riede, M. D. McGehee, D. Neher, A. Salleo, Nat. Mater. 2014, 13, 63.

[44] S. M. Tuladhar, D. Poplavskyy, S. A. Choulis, J. R. Durrant, D. D. C. Bradley, J. Nelson, Adv. Funct. Mater. 2005, 15, 1171.

[45] A. Gadisa, K. Tvingstedt, K. Vandewal, F. Zhang, J. V. Manca, O. Inganäs, Adv. Mater. 2010, 22, 1008.

[46] R. Noriega, J. Rivnay, K. Vandewal, F. P. V. Koch, N. Stingelin, P. Smith, M. F. Toney, A. Salleo, Nat. Mater. 2013, 12, 1038.

[47] F. Symalla, P. Friederich, A. Massé, V. Meded, R. Coehoorn, P. Bobbert, W. Wenzel, Phys. Rev. Lett. 2016, 117, 276803. 
a

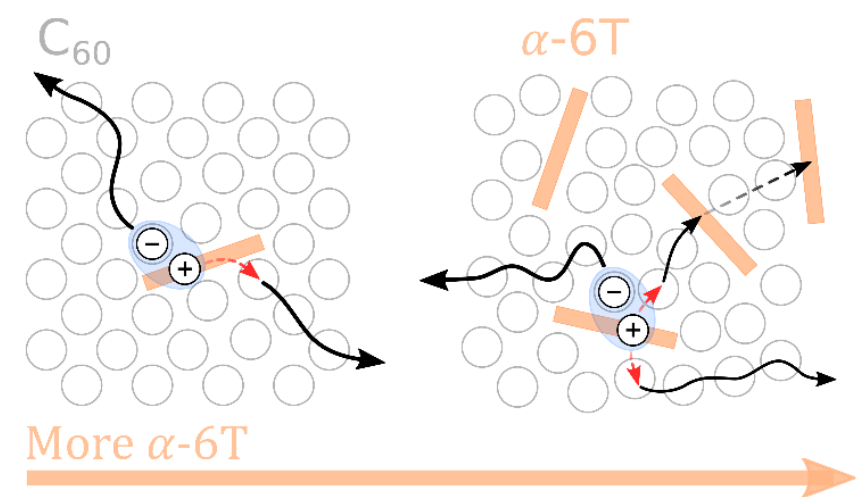

Low $F$
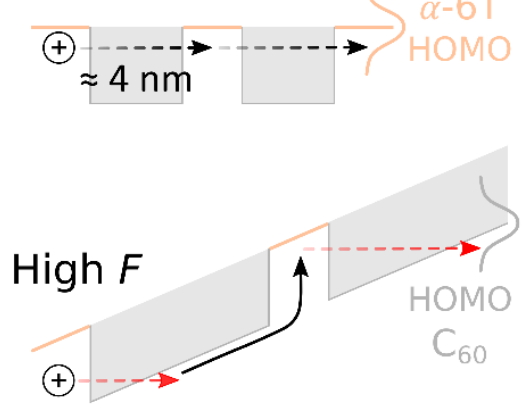

b

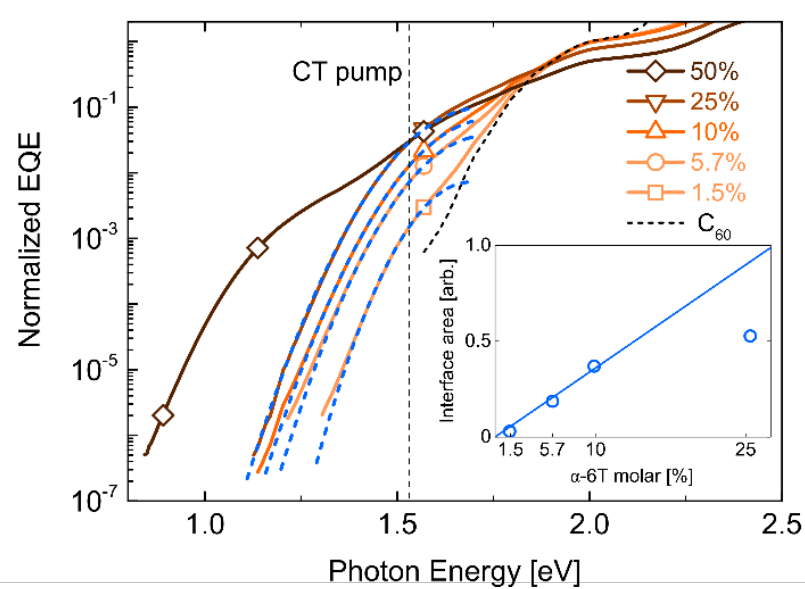

C

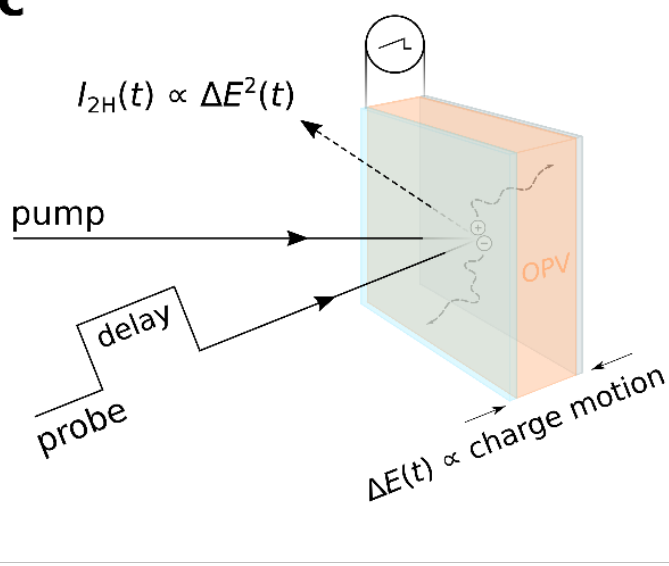

Figure 1 | Schematic representation of charge transport in the investigated OPV devices, their EQE spectra and a scheme of the transient measurement technique. (a) With increasing donor content the distance between isolated $\alpha$-6T decreases but the $\mathrm{C}_{60}$ matrix gets increasingly disordered. Electron or hole transport in $\mathrm{C}_{60}$ is indicated by solid black arrows. Dashed red arrows indicate hole de-trapping from $\alpha$-6T to $\mathrm{C}_{60}$ by Fowler-Nordheim-type tunneling (only occurs at high electric fields $F$ ), whereas black dashed arrows indicate long-range hole tunneling through $\mathrm{C}_{60}$ to nearby $\alpha-6 \mathrm{~T}$ sites. Sites are spread according to a Gaussian DOS distribution as indicated. (b) Sensitive EQE measurements. The inset shows the strength of CT absorption as inferred from Gaussian fitting to the CT state manifold (dashed blue traces), see the main text. (c) Schematic description of the combined TREFISH and photocurrent experiment. 


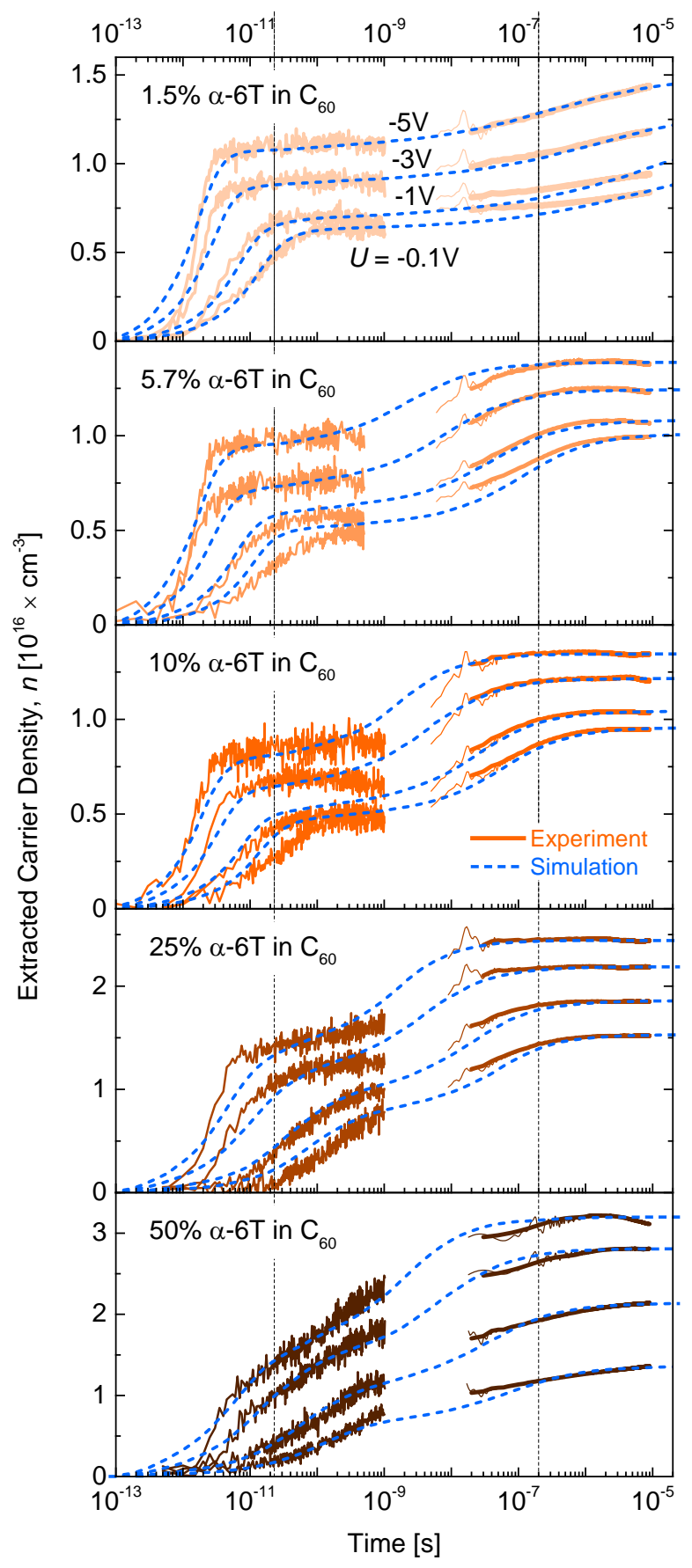

Figure 2 | Time-resolved extraction of photo-generated charges. Experiments (colored traces) at the indicated applied reverse bias $U$ and least-square fits by the extended Gaussian disorder model (blue dashed traces) involving three charge carrier populations: electrons in $\mathrm{C}_{60}$, holes in $\mathrm{C}_{60}$ and holes tunneling through $\mathrm{C}_{60}$ between isolated $\alpha$-6T sites. Increasing orange color saturation indicates increasing $\alpha$-6T molar fraction (from top to bottom). 

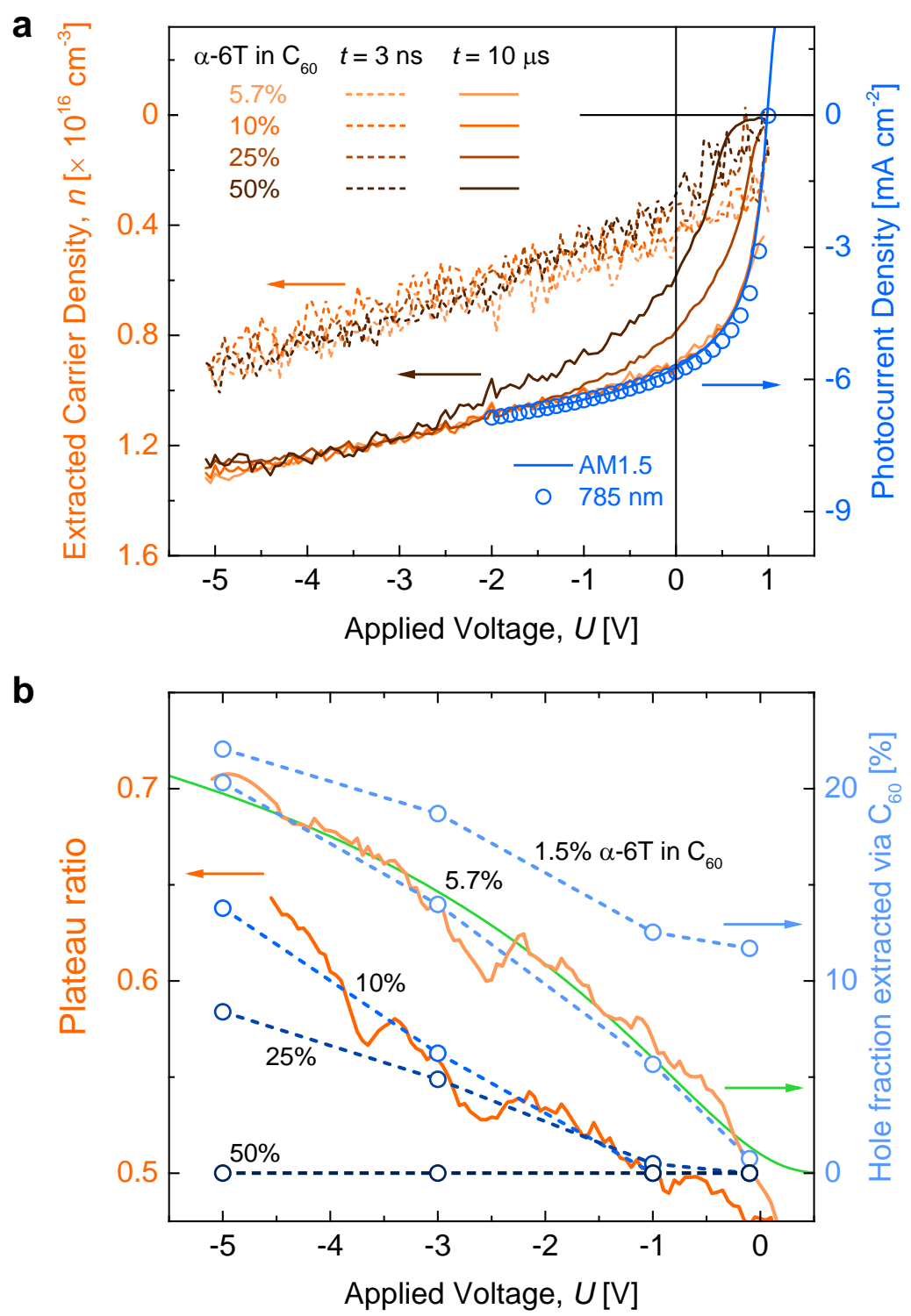

Figure 3 | Photocurrent-voltage characteristics and the fraction of photo-generated holes extracted via $\mathbf{C}_{\mathbf{6 0}}$. (a) Steady-state photocurrent-voltage measurements under AM1.5 illumination (blue trace, corrected by the dark IV, 10\% $\alpha$-6T device, SI Figure S3 shows other devices), steady-state photocurrent-voltage measurements under continuous $785 \mathrm{~nm}$ laser illumination (blue symbols, corrected by the dark IV, scaled to AM1.5 data, scaling factors shown in SI Figure S3) and the total collected charge recorded by pulsed measurements (solid orange traces $t=10 \mu \mathrm{s}$ ). Dashed orange traces indicate the total photo-generated charge extracted at $t=3 \mathrm{~ns}$. (b) Orange traces indicate the plateau ratio in extracted carrier density for the $5.7 \%$ and $10 \% \alpha$-6T devices in Figure 2. Higher ratios than 0.5 indicate that a fraction of holes is extracted via $\mathrm{C}_{60}$ (right axis). Blue traces are the estimated hole fraction from the model fits to experiment in Figure 2. The green trace is a fit to Fowler-Nordheim-type tunneling. ${ }^{[32]}$ 

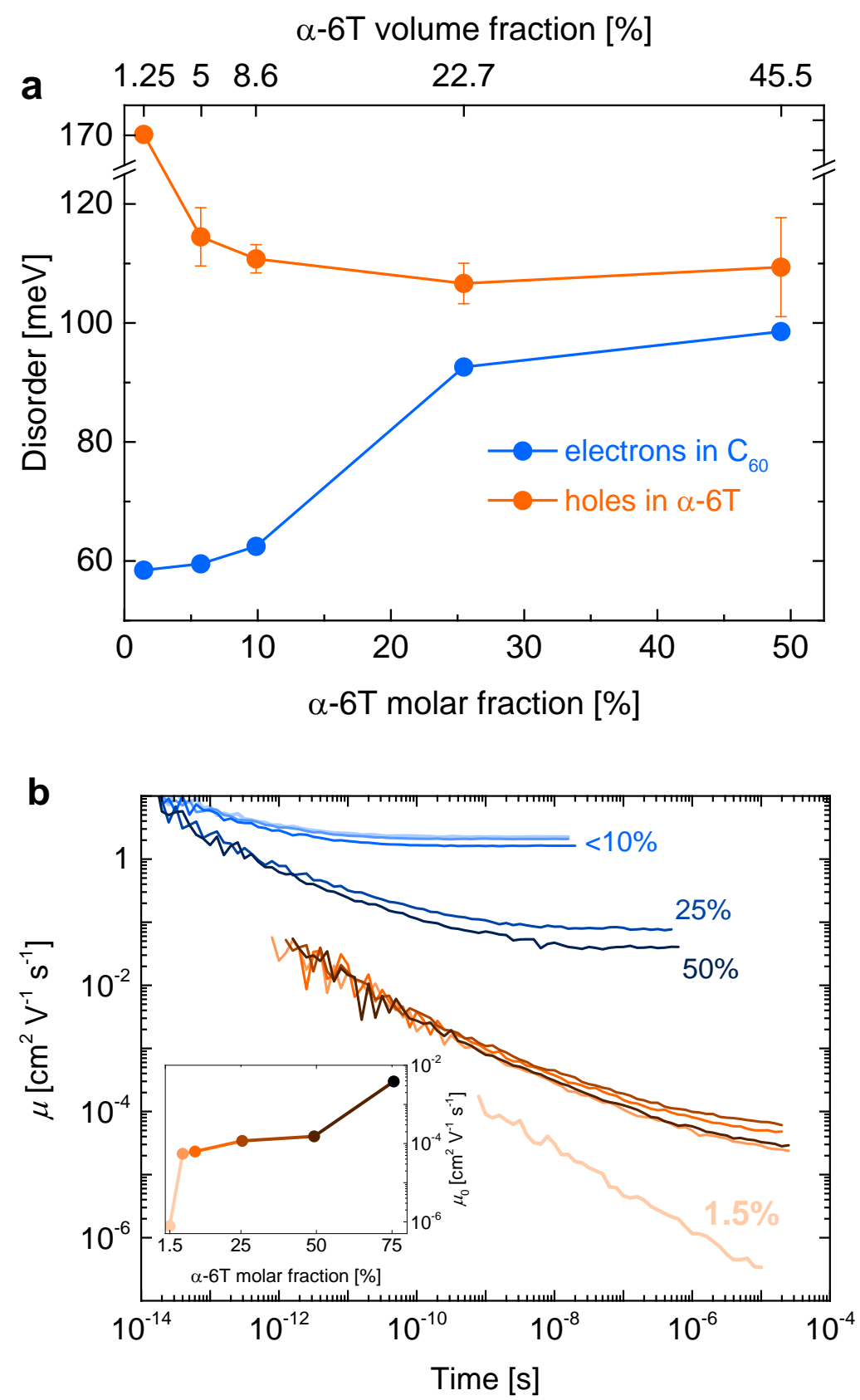

Figure 4 | Energetic disorder and photo-generated carrier mobility with increasing $\alpha-6$ T content. (a) Electron (blue symbols) and hole (orange symbols) energetic disorder of the Gaussian DOS versus $\alpha$-6T content. Hole disorder was estimated both from transient and SCLC measurements, symbols indicate the mean, whereas errors bars indicate the corresponding standard error. (b) Transient mobility of photo-generated holes (orange traces) and electrons (blue traces). The inset shows the experimental steady-state SCLC hole mobility $\mu_{0}$ in the low carrier-density regime (see the Experimental Section), the increase in $\mu_{0}$ at $1.5-5.7 \%$ marks the onset for efficient long-range hole tunneling, whereas the increase at 50-75\% marks the formation of a continuous donor network. 


\section{Charge Transport in Pure and Mixed Phases in Organic Solar Cells}

Armantas Melianas*, Vytenis Pranculis, Donato Spoltore, Johannes Benduhn, Olle Inganäs, Vidmantas Gulbinas, Koen Vandewal, Martijn Kemerink*

Email: - ${ }^{*}$ Armantas.Melianas@liu.se

-3artijn.Kemerink@liu.se

\section{Contents}

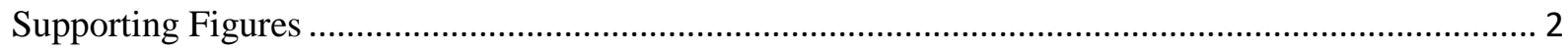

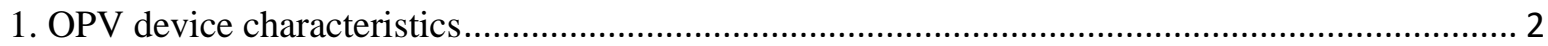

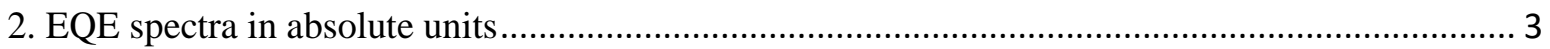

3. Comparison of photocurrent-voltage curves at different illumination conditions........................... 4

4. Transient measurements were performed in the linear pump-fluence regime ............................... 5

5. Hole-only diode SCLC characteristics and the estimated hole mobility ..................................... 6

6. Fowler-Nordheim-type tunneling at high fields ......................................................................... 7

7. Kinetic Monte Calro Simulations taking into account the active layer morphology ...................... 8

8. Increase of energetic disorder in $\mathrm{C}_{60}$ with increasing donor content …..................................... 9

9. Experimental estimate for the hole localization length .............................................................. 10

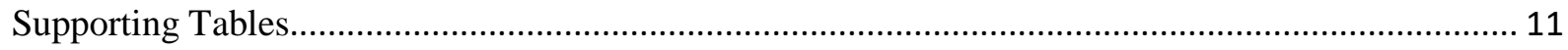

10. Weight, molar and volume fractions and estimated distance between isolated $\alpha-6 \mathrm{~T} \ldots \ldots \ldots \ldots \ldots . . . .11$

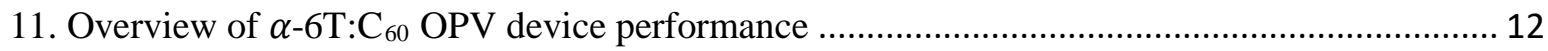

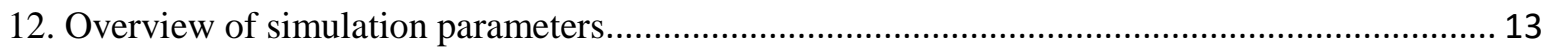

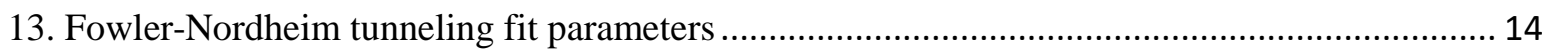

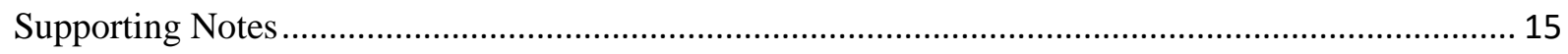

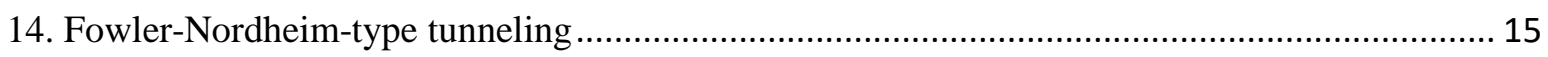

15. Tunneling through a rectangular barrier and the effective mass of the tunneling hole ............... 17

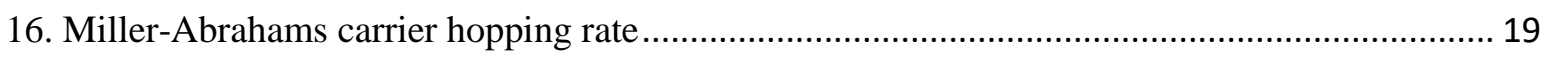

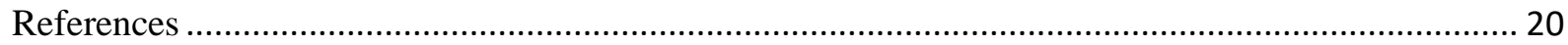




\section{Supporting Figures}

\section{OPV device characteristics}
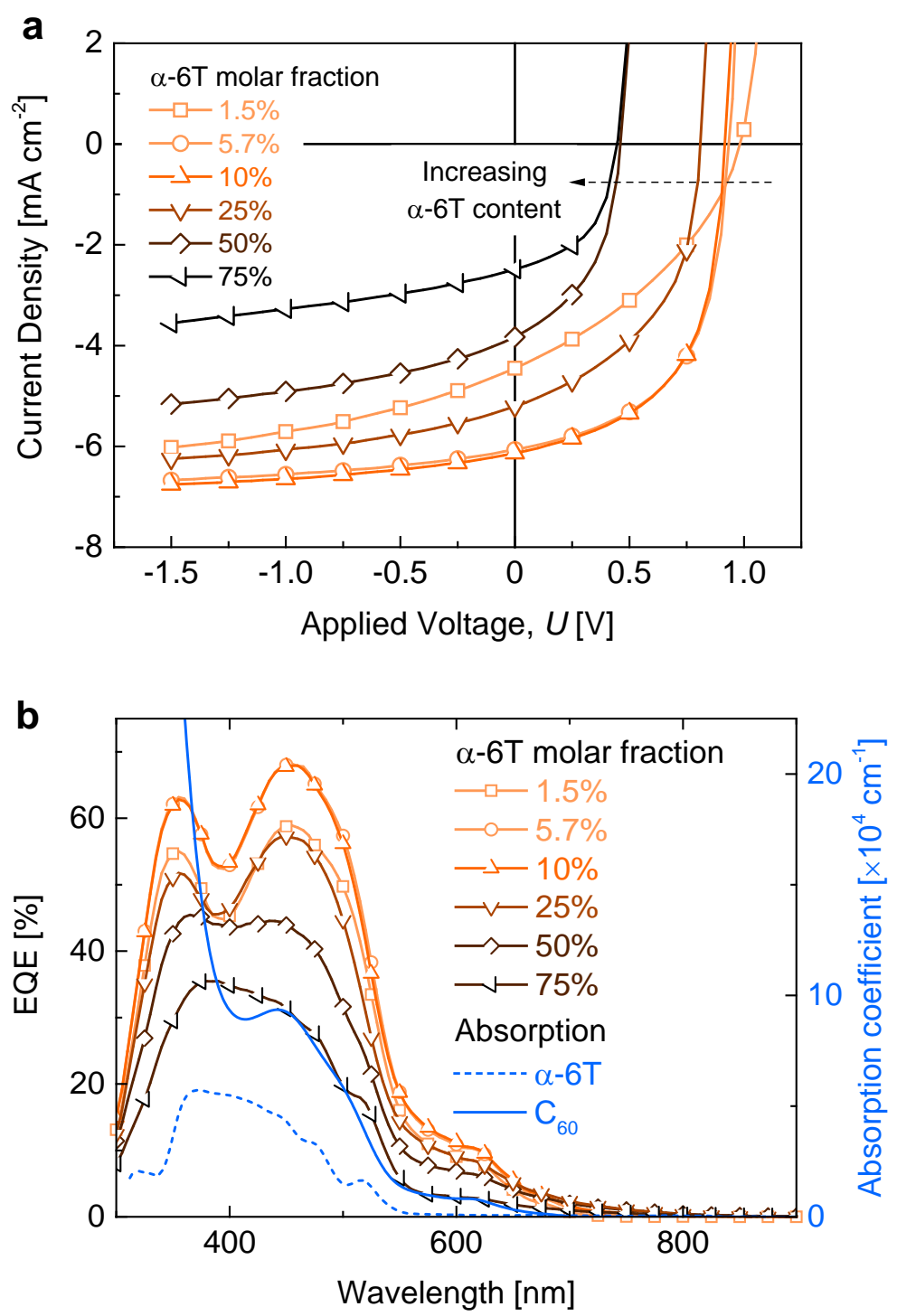

Figure S1 | OPV device characteristics. (a) Steady-state IV characteristics under AM1.5 illumination of $\alpha-6 \mathrm{~T}: \mathrm{C}_{60}$ OPV devices with the indicated molar fraction of $\alpha$-6T. Device area is larger than that of OPV devices used in the transient measurements $\left(6.44 \mathrm{~mm}^{2}\right.$ vs $\left.1.68 \mathrm{~mm}^{2}\right)$, nevertheless the devices are nominally identical, see Table S2. (b) EQE spectra of the corresponding OPV devices and the absorption coefficients of the constituent materials, determined by spectroscopic ellipsometry. Note that the $J_{\mathrm{SC}}$ of these devices is mainly limited by the narrow absorption of $\mathrm{C}_{60}$ (blue trace) and the even lower absorption of the high optical gap $\alpha-6 \mathrm{~T}$ (dashed blue trace). Both molecules were chosen on purpose as outlined in the main text - the limited $J_{\mathrm{SC}}$ and PCE are not so important. Most importantly, we can obtain an $\mathrm{EQE}=55-70 \%$ and $F F=0.55-0.57$ in the absorbing range, see Table S2. 


\section{EQE spectra in absolute units}

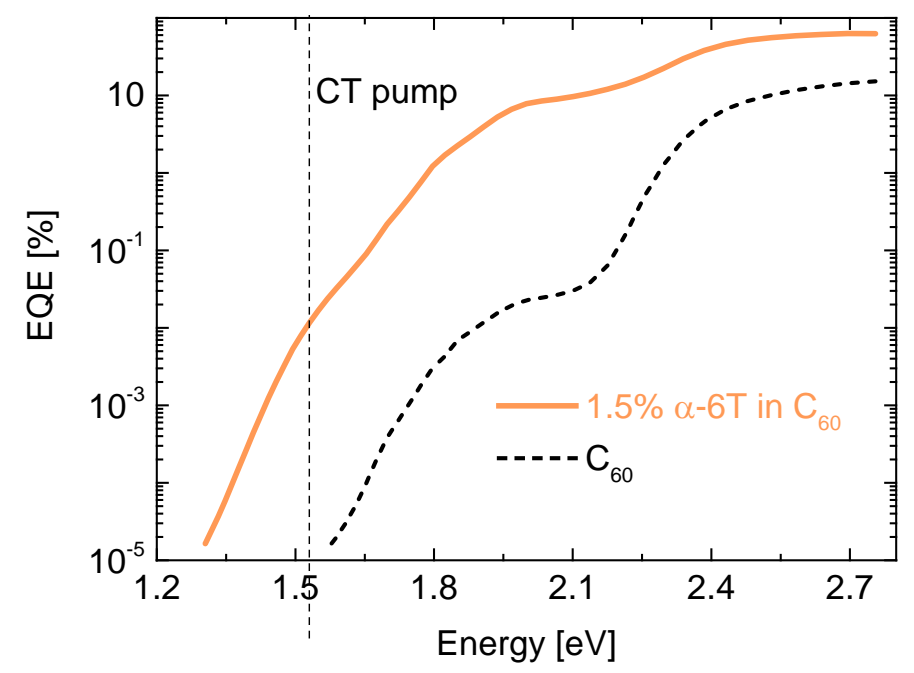

Figure S2 | EQE spectra in absolute units. At the excitation wavelength used in the transient measurements $810 \mathrm{~nm}(1.53 \mathrm{eV})$ the number of extracted charge carriers photo-generated in neat $\mathrm{C}_{60}$ is much less compared to the number of extracted charge carriers photo-generated in the $1.5 \% \alpha$-6T device. We only show the data for the $1.5 \% \alpha-6 \mathrm{~T}$ device as the contribution from the CT manifold is even stronger with increasing $\alpha-6 \mathrm{~T}$ content, see Figure $1 \mathrm{~b}$ of the main text. Due to the low pump-photon energy $(1.53 \mathrm{eV})$ we do not expect a significant increase in the bulk ionization efficiency in $\mathrm{C}_{60}$ even at high reverse bias $(U=-5 \mathrm{~V})$. As such, at the excitation energy used in the transient measurements $(1.53 \mathrm{eV})$ the charge carriers are photo-generated in CT states. 


\section{Comparison of photocurrent-voltage curves at different illumination conditions}
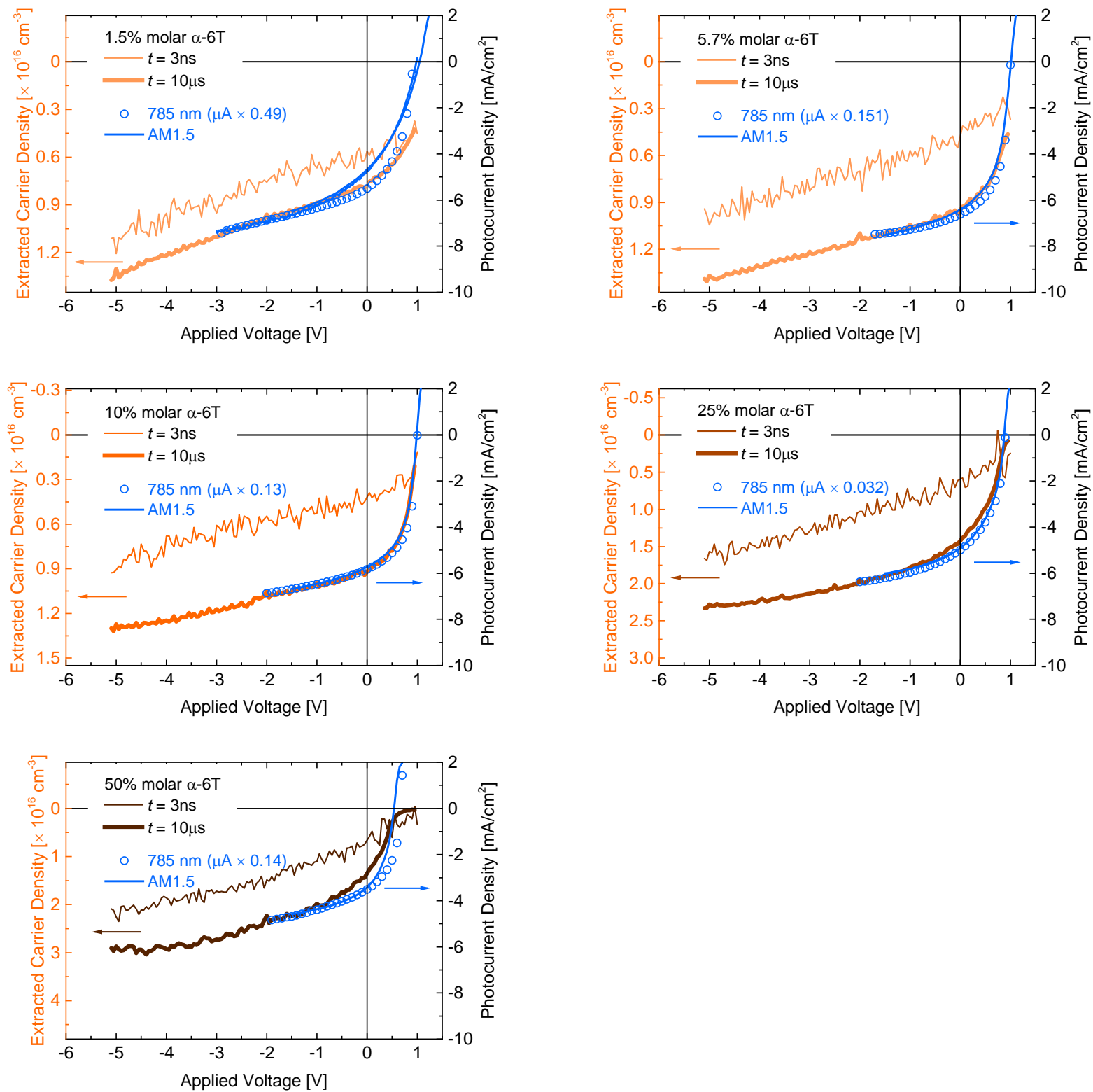

Figure S3 | Comparison of photocurrent-voltage curves measured using pulsed $810 \mathrm{~nm}$ excitation (orange traces), continuous AM1.5 illumination (blue lines) or continuous illumination by a $785 \mathrm{~nm}$ laser (blue symbols). In case of continuous illumination the dark IVs were subtracted from IVs under illumination, so the blue lines and symbols indicate the device photocurrent. Photocurrent vs V from transient measurements were estimated by recording the total amount of collected charge at $t=10 \mu$ s versus applied bias. In case of pulsed excitation the correction in the dark is not necessary as it is implicitly included in the transient measurement (with/without pump = light/dark). Reasonably good agreement is obtained among the different excitations conditions. The data collected under continuous illumination by a $785 \mathrm{~nm}(1 \mathrm{~mW})$ laser were linearly scaled for comparison with the AM1.5 photocurrent trace, the scaling factors are indicated in the Figure. 


\section{Transient measurements were performed in the linear pump-fluence regime}
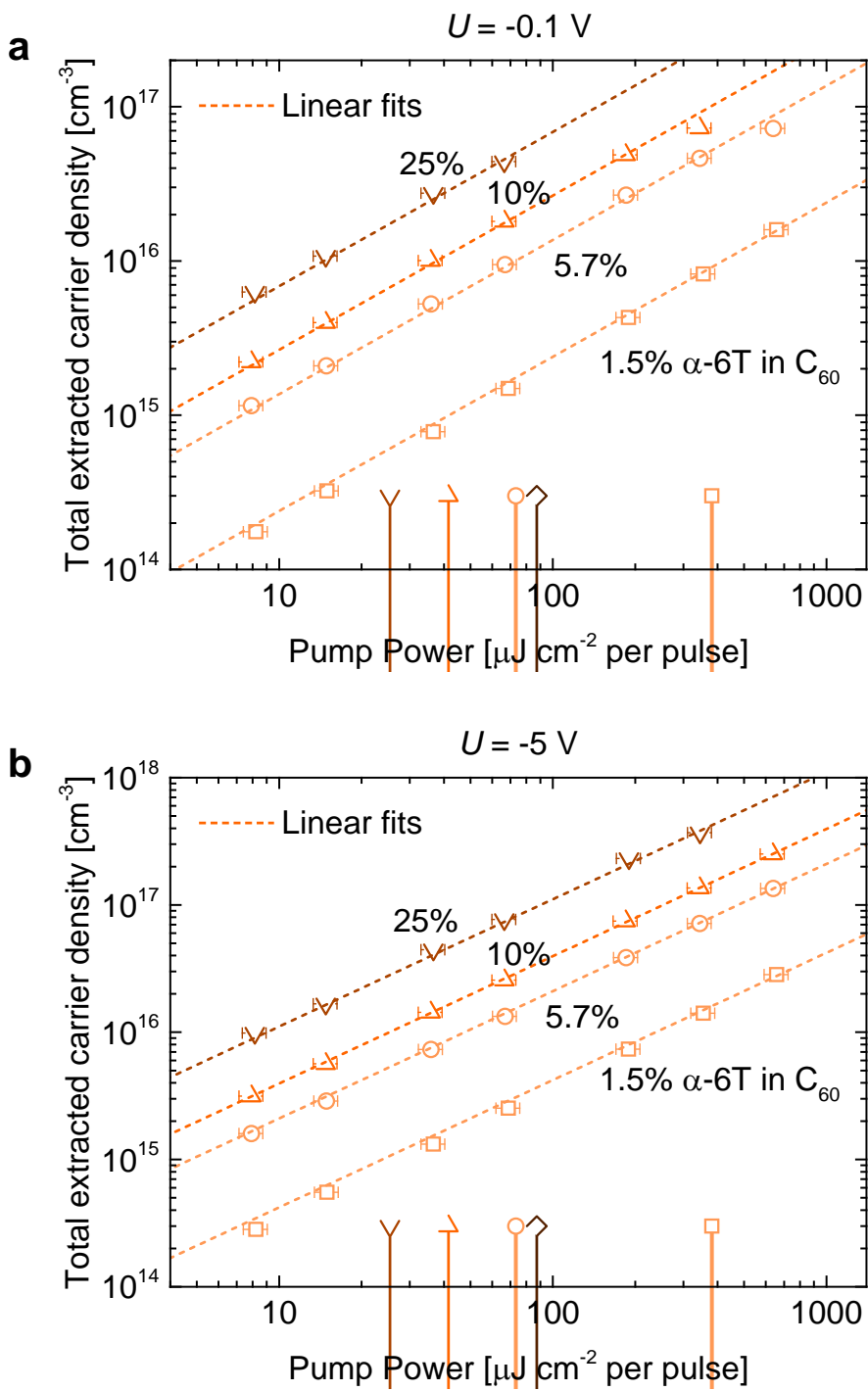

Figure S4 | Transient measurements were performed in the linear pump-fluence regime. The total extracted carrier density from the OPV device (symbols) scales linearly (dashed traces) with the indicated pump power per pulse (810 nm pump) at both (close to) short-circuit conditions $(U=-0.1 \mathrm{~V})(\mathbf{a})$ and high reverse bias $(U=-5 \mathrm{~V})(\mathbf{b})$. Note that CT absorption is orders of magnitude weaker than absorption of the constituent materials. As such, the pump-fluences used in this study may be considered as relatively low. Vertical lines at the bottom of the Figure indicate the pump-fluence used in the transient measurements of the main text. The pump-fluence used in the transient measurements for the $50 \%$ device $\left(87.4 \mu \mathrm{J} \mathrm{cm}^{-2}\right.$ per pulse) is indicated by the vertical line with a diamond symbol, which is comparable to the pump-fluence used for the $5.7 \%$ device $\left(73.2 \mu \mathrm{J} \mathrm{cm}^{-2}\right.$ per pulse). On basis of the observed linear trend in other samples and the rather low pump-fluence chosen for transient measurements, the pump-fluence dependence was not recorded for the $50 \%$ device, as it is also expected to be in the linear regime. Since all transient measurements were performed in the linear regime, this allows us to scale the transient data for comparison, such as in Figure 3a of the main text and Figure S3. 


\section{Hole-only diode SCLC characteristics and the estimated hole mobility}
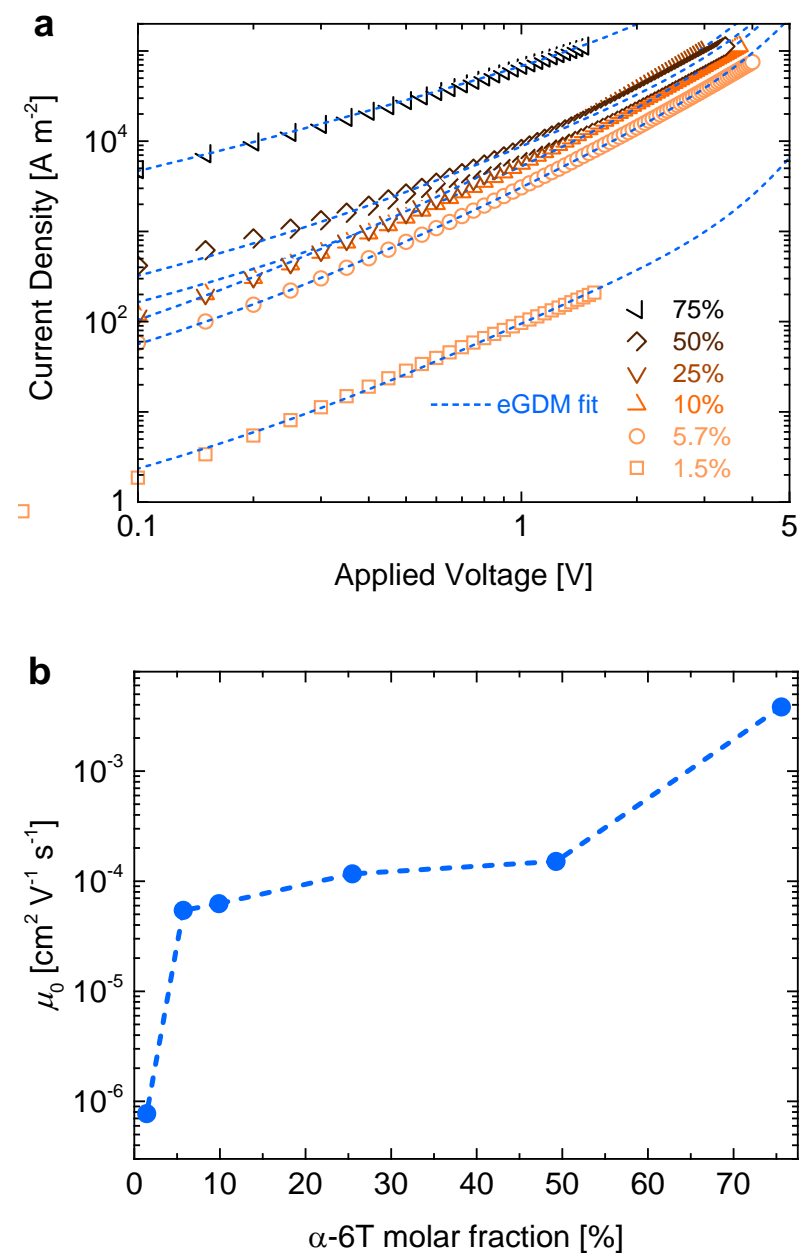

Figure S5 | Hole-only diode SCLC characteristics and the estimated hole mobility of BHJs with the indicated molar fraction of $\alpha-6 \mathrm{~T}$ in a $\mathrm{C}_{60}$ matrix. (a) The SCLC data were fitted by very similar charge carrier hopping parameters as those used for the transient measurements, see Table S3, to ensure that both transient and steady-state charge transport in these blends is well characterized by the model. (b) Steady-state SCLC hole mobility $\mu_{0}$ in the low-concentration regime (fractional DOS occupancy of $c_{0}=10^{-4}$ at $300 \mathrm{~K}$ and an electric field strength of $0.5 \mathrm{~V}$ per $50 \mathrm{~nm}$, as in transient mobility simulations, were used to estimate $\mu_{0}$ according to the parametrization by Pasveer $\left.{ }^{[1]}\right)$. The increase in $\mu_{0}$ at $1.5-5.7 \%$ marks the onset for efficient long-range hole tunneling, whereas the increase at $75 \%$ possibly marks the formation of a continuous donor network, as can also be inspected from the approximately 40-fold increase in the attempt-to-hop frequency of the holes, see Table S3. 


\section{Fowler-Nordheim-type tunneling at high fields}

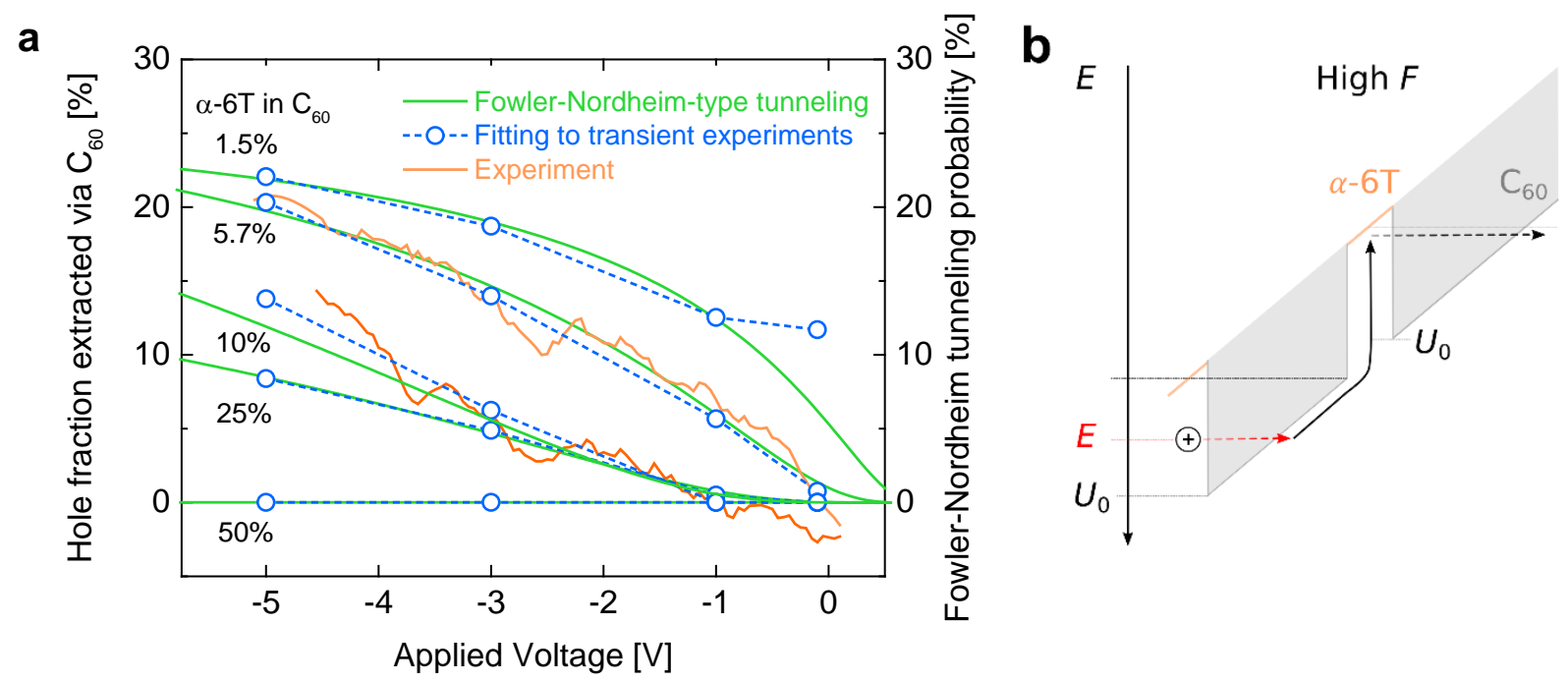

Figure S6 | Fowler-Nordheim-type tunneling. (a) The estimated fraction of holes that undergo de-trapping from $\alpha-6 \mathrm{~T}$ to $\mathrm{C}_{60}$ in experiment (orange traces) and from model fits to transient experiments (blue traces) follow the $\propto \exp (-1 / F)$ field-dependence, as analytically derived by Fowler and Nordheim for particle tunneling through a triangular energy barrier (green traces). See Note S1 for the Fowler-Nordheim equation describing the tunneling probability and Table S4 for the fit parameters. As the precise details of tunneling may vary significantly between different materials and situations, and since a detailed derivation is outside the scope of this article, here we limit ourselves to only the necessary analysis to explain the experiments. (b) In principle, the tunneling probability through a triangular energy barrier of a particle with excess energy $E$ (such as directly after photo-excitation) is higher than that of a particle tunneling from the lowest energy of the trap site (such as after a prolonged trapping event), compare the red and the black dashed arrows in the schematic (Notes S1 and S2 contain additional discussion). We cannot distinguish the two situations in experiment. Nevertheless, since the field-dependence of the tunneling probability is the same in both cases (see Note S1), the field-dependence for hole de-trapping to $\mathrm{C}_{60}$ observed in experiment can be explained by the same Fowler-Nordheim-type tunneling process. Note that with bias the shape of the tunneling barrier will gradually change from a triangle (at high reverse bias) to a trapezoid (intermediate to low reverse bias) and then to a rectangle (at low reverse bias relevant to OPV devices). A similar transition at moderate bias $(<1 \mathrm{~V})$ has been demonstrated by Kushmerick et al. in molecular junctions of $\pi$-conjugated thiols. ${ }^{[2]}$ Our measurement resolution is insufficient to observe this transition. However, as the measured fraction of de-trapped holes goes to zero at fields relevant to OPV devices (short-circuit to $V_{\text {OC }}$, corresponding to an applied voltage of $0 \mathrm{~V}$ to $\approx 1 \mathrm{~V}$ ), tunneling between isolated donor sites in operating OPV devices is expected to occur through a rectangular energy barrier. The tunneling probability through a rectangular energy barrier is field-independent and mainly limited by $U_{0}$ (donor and $\mathrm{C}_{60}$ HOMO level difference) and the barrier width (distance between donor sites), see Note S2. 


\section{Kinetic Monte Calro Simulations taking into account the active layer morphology}

a

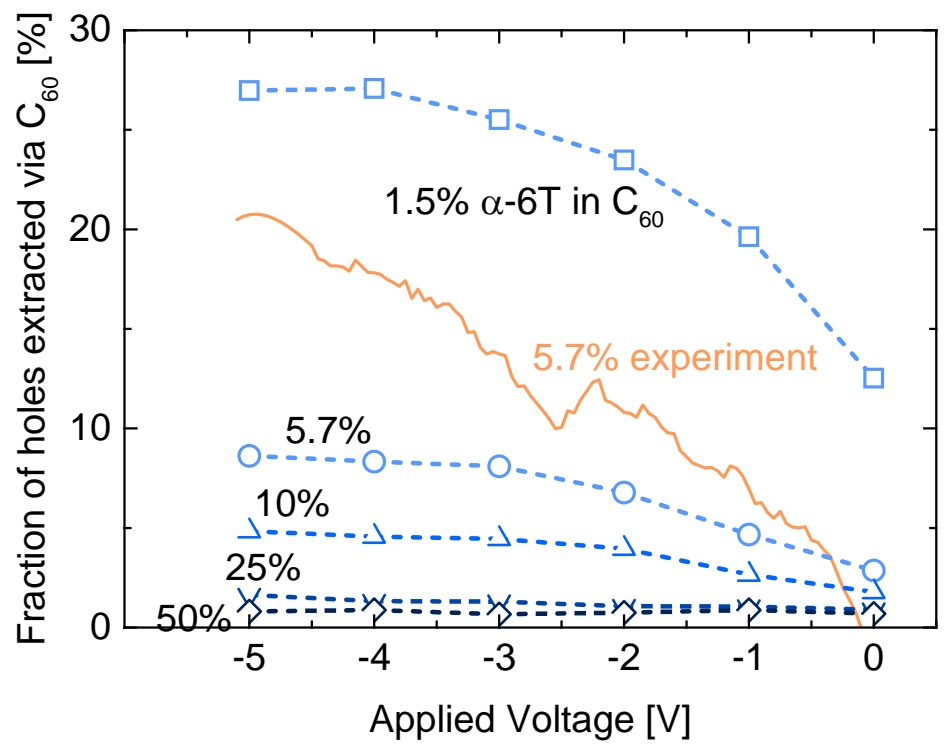

b

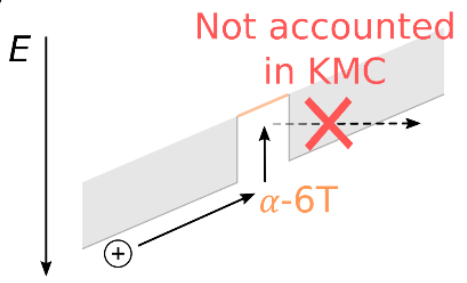

High $F$

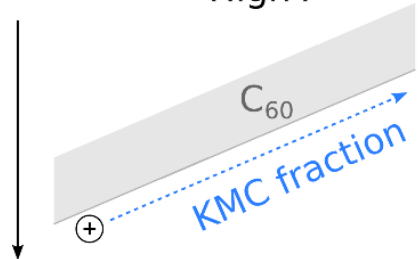

Figure S7 | Kinetic Monte Carlo Simulations taking into account the active layer morphology. (a) Kinetic Monte Carlo (KMC) simulations confirm the trend observed in experiments and obtained from model fits to experiments, see Figure $3 \mathrm{~b}$ of the main text. These more advanced simulations were performed by taking into account the 3D morphology of the BHJ active layer: a fraction of the total simulated volume 90x90x50 (nm $\left.{ }^{3}\right)$ was occupied by randomly dispersed $\alpha$-6T sites using the known donor volume fractions. The energy barrier for hole de-trapping from $\alpha$-6T to $\mathrm{C}_{60}$ was also fixed to that known from experiments $(0.9 \mathrm{eV})$. Photo-generated holes and electrons were initially assumed to reside in $\mathrm{C}_{60}$, which was done purposely to allow us to quantify the fraction of holes that are extracted without a single trapping event in isolated $\alpha$-6T. Hole and electron motion was described by the carrier hopping parameters obtained from the model fits to experiments in Figure 2 of the main text, see Table S3. KMC simulations suggest that there is a fast fraction of holes $(0-30 \%$ of the total in this case, depending on bias) that is extracted via $\mathrm{C}_{60}$ without a single trapping event in isolated $\alpha$-6T. The remaining hole fraction ( $0-70 \%$ of the total in this case) undergoes trapping in $\alpha-6 \mathrm{~T}$, in which case further hole motion requires either tunneling through $\mathrm{C}_{60}$ to a neighboring donor site or a large electric field to allow for Fowler-Nordheim-type de-trapping back to $\mathrm{C}_{60}$ (Figure S6). Both of these events are not taken into account in the KMC simulations on purpose (see panel b). This is why at donor content of 5.7\% molar and higher, the fractions obtained from experiments (orange trace in panel a, see also Figure $3 b$ of the main text) are found to be larger than predicted by the KMC model. Therefore, the fast fraction of holes (0-20\% of the total, orange trace) consists of holes extracted via $\mathrm{C}_{60}$ without a single trapping event ( $0-10 \%$ of the total, blue trace at $5.7 \%$ molar) and holes captured by $\alpha-6 \mathrm{~T}$ but undergoing ultra-fast Fowler-Nordheim-type de-trapping back to $\mathrm{C}_{60}$ (possible only at high fields $F$ ). (b) Schematic describing the fraction of holes shown in panel a. 


\section{Increase of energetic disorder in $\mathrm{C}_{60}$ with increasing donor content}
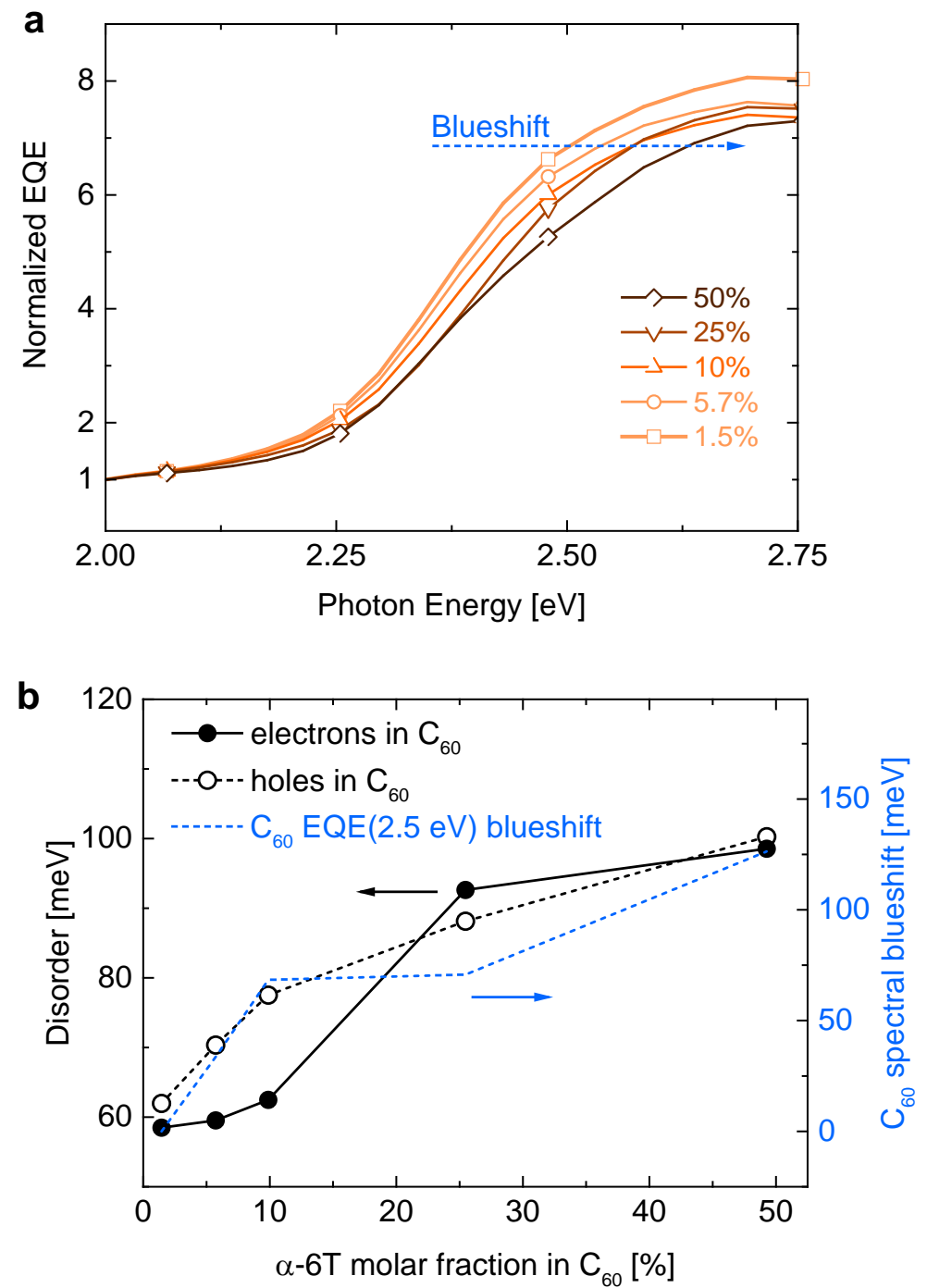

Figure S8 | Increase of energetic disorder in $\mathbf{C}_{60}$ with increasing donor content. (a) Zoomed-in portion of the EQE spectra shown in the main text (Figure 1b), normalized to the $\mathrm{C}_{60}$ absorption peak at $2.0 \mathrm{eV}$ for comparison. $2.0 \mathrm{eV}$ corresponds to an intra-molecular singlet absorption of $\mathrm{C}_{60}{ }^{[3]}$ and is thus morphology-independent, whereas $2.5 \mathrm{eV}$ corresponds to an inter-molecular charge-transfer absorption of $\mathrm{C}_{60}{ }^{[3]}$ and thus depends on the disorder of the $\mathrm{C}_{60}$ phase. The spectral blueshift at $2.5 \mathrm{eV}$ relative to the $1.5 \% \alpha-6 \mathrm{~T}$ sample is highlighted by the dashed blue arrow. The relative blueshift is estimated by following a line of constant EQE, which is equal to that of the $1.5 \% \alpha-6 \mathrm{~T}$ sample at $2.5 \mathrm{eV}$. Note that, although the blueshift is expected to be dominated by the absorption in $\mathrm{C}_{60}$, also $\alpha-6 \mathrm{~T}$ weakly absorbs at $2.5 \mathrm{eV}$ (Figure S1) and may contribute to the blueshift in the spectra. Moreover, the indicated blueshift depends on the photon energy at which it is estimated. As such, the indicated blueshift is only indicative for the formation of more amorphous $\mathrm{C}_{60}$ and should not be considered as an accurate estimate. (b) Comparison of the increasing electron and hole disorder in $\mathrm{C}_{60}$ (obtained by the eGDM fits to transient experiments) to the spectral blueshift of the EQE spectra described in panel (a). 


\section{Experimental estimate for the hole localization length}

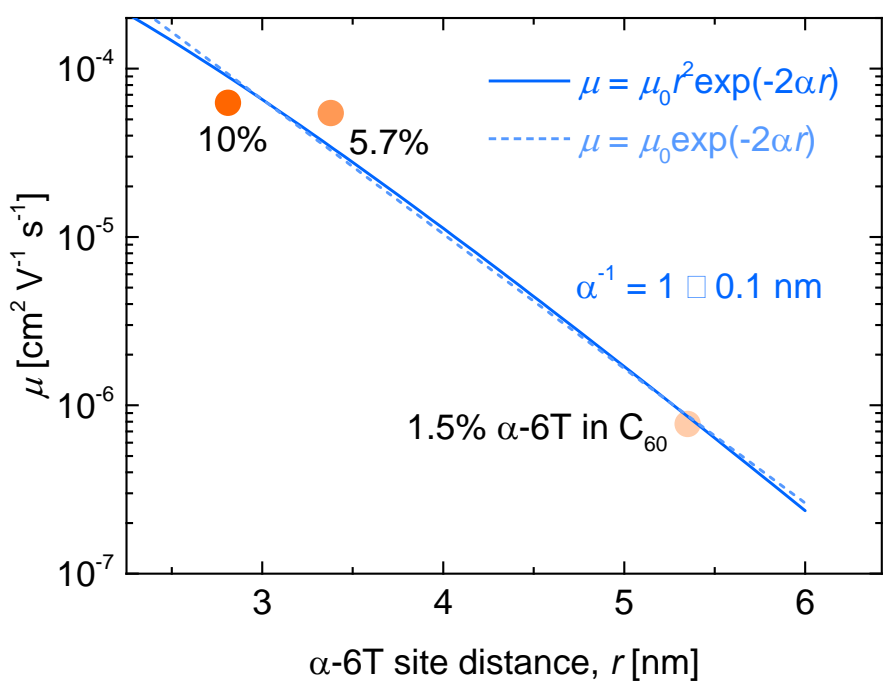

Figure S9 | Experimental estimate for the hole localization length using SCLC data. Hole-only SCLC mobility data (symbols, data estimated for $c_{0}=10^{-4}$ at $300 \mathrm{~K}$ and an electric field strength of $0.5 \mathrm{~V}$ per $50 \mathrm{~nm}$, using the parametrization by Pasveer ${ }^{[1]}$ ) of samples with a homogeneously diluted donor ( $<10 \%$ molar) allow us to estimate the hole localization length $\alpha^{-1}$ from SCLC experiments. This parameter describes the effective wavefunction overlap between localized sites in most charge transport models, typically via one of the two equations $\mu=\mu_{0} r^{2} \exp (-2 \alpha r)$ or $\mu=\mu_{0} \exp (-2 \alpha r)$, highlighted in the Figure. We obtain good fits (blue lines) in both cases, estimating the hole localization length to be in the range $\alpha^{-1}=1 \pm 0.2 \mathrm{~nm}$. This is a surprisingly large number as typically $\alpha^{-1} \sim 0.1 \mathrm{~nm}$ is assumed, ${ }^{[1,4]}$ based on earlier data for for TNF:PVK $\left(\alpha^{-1} \approx 0.11 \mathrm{~nm}\right)$ by Gill ${ }^{[5]}$ and for P3HT and $\mathrm{OC}_{1} \mathrm{C}_{10}-\mathrm{PPV}$ $\left(\alpha^{-1} \approx 0.15 \mathrm{~nm}\right.$ ) by de Leeuw et al. in ref. [6]. Full material names are given in the Experimental Section. For large $\alpha^{-1}$ the hole wavefunction extends a larger distance from the donor site, enabling long-range tunneling. 


\section{Supporting Tables}

\section{Weight, molar and volume fractions and estimated distance between isolated $\alpha-6 \mathrm{~T}$}

\begin{tabular}{ccccc}
\hline $\begin{array}{c}\text { Donor-acceptor } \\
\text { stoichiometry }\end{array}$ & $\begin{array}{c}\alpha \text {-6T weight } \\
\text { fraction [\%] }\end{array}$ & $\begin{array}{c}\alpha \text {-6T volume } \\
\text { fraction [\%] }\end{array}$ & $\begin{array}{c}\alpha \text {-6T molar } \\
\text { fraction [\%] }\end{array}$ & $\begin{array}{c}\text { Distance between } \alpha \text {-6T } \\
\text { sites [nm] }\end{array}$ \\
\hline $1: 99$ & 1 & 1.25 & $1.45(\approx 1.5)$ & 5.35 \\
4:96 (1:24) & 4 & 4.95 & $5.72(\approx 5.7)$ & 3.38 \\
$7: 93(1: 13.3)$ & 7 & 8.61 & $9.88(\approx 10)$ & 2.81 \\
19:81 (1:4.26) & 19 & 22.69 & $25.47(\approx 25)$ & 2.03 (possibly unreliable) \\
40:60 (2:3) & 40 & 45.48 & $49.27(\approx 50)$ & 1.61 (not applicable) \\
$68: 32(2.125: 1)$ & 68 & 72.67 & $75.58(\approx 75)$ & 1.38 (not applicable) \\
\hline
\end{tabular}

Table S1. The volume fraction of $\alpha$-6T was estimated using the mass density of $\alpha$-6T $\left(1.4 \mathrm{~g} \mathrm{~cm}^{-3}\right)$ and $\mathrm{C}_{60}\left(1.63 \mathrm{~g} \mathrm{~cm}^{-3}\right)$, whereas the corresponding molar fraction was estimated using the molar density of $\alpha-6 \mathrm{~T}\left(494.736 \mathrm{~g} \mathrm{~mol}^{-1}\right)$ and $\mathrm{C}_{60}\left(720.66 \mathrm{~g} \mathrm{~mol}^{-1}\right)$. The distance between $\alpha$-6T sites was approximated as twice the radius of a sphere containing an estimated volume fraction of $\mathrm{C}_{60}$ and an isolated $\alpha$-6T in the center of the sphere. Due to the elongated shape of the $\alpha$-6T molecule and its strong tendency to aggregate, this estimate is expected to be a reasonable upper limit only for $\alpha$-6T content up to $10 \%$ molar. $\mathrm{C}_{60}$ diameter is $0.71 \mathrm{~nm}$ (center-to-center distance between $\mathrm{C}_{60}$ molecules is $\approx 1 \mathrm{~nm}$ ). 
11. Overview of $\alpha-6 \mathrm{~T}: \mathrm{C}_{60} \mathrm{OPV}$ device performance

\begin{tabular}{cccccc}
\hline $\begin{array}{c}\text { Device area } \\
{\left[\mathrm{cm}^{2}\right]}\end{array}$ & $\begin{array}{c}\alpha-6 \mathrm{~T} \text { molar } \\
{[\%]}\end{array}$ & $J_{\mathrm{SC}}\left[\mathrm{mA} \mathrm{cm}^{-2}\right]$ & $V_{\mathrm{OC}}[\mathrm{V}]$ & $F F$ & PCE [\%] \\
\hline $1.68 \mathrm{~mm}^{2}$ & 1.5 & 4.67 & 0.97 & 0.379 & 1.68 \\
$1.68 \mathrm{~mm}^{2}$ & 5.7 & 6.13 & 0.93 & 0.530 & 3.09 \\
$1.68 \mathrm{~mm}^{2}$ & 10 & 5.86 & 0.91 & 0.567 & 2.99 \\
$1.68 \mathrm{~mm}^{2}$ & 25 & 5.21 & 0.81 & 0.493 & 2.06 \\
$1.68 \mathrm{~mm}^{2}$ & 50 & 3.60 & 0.46 & 0.482 & 0.78 \\
$1.68 \mathrm{~mm}^{2}$ & 75 & 2.38 & 0.45 & 0.491 & 0.53 \\
$6.44 \mathrm{~mm}^{2}$ & 1.5 & 4.45 & 0.98 & 0.365 & 1.62 \\
$6.44 \mathrm{~mm}^{2}$ & 5.7 & 6.07 & 0.93 & 0.551 & 3.18 \\
$6.44 \mathrm{~mm}^{2}$ & 10 & 6.14 & 0.92 & 0.574 & 3.17 \\
$6.44 \mathrm{~mm}^{2}$ & 25 & 5.40 & 0.81 & 0.458 & 1.98 \\
$6.44 \mathrm{~mm}^{2}$ & 50 & 3.83 & 0.46 & 0.477 & 0.82 \\
$6.44 \mathrm{~mm}^{2}$ & 75 & 2.48 & 0.45 & 0.494 & 0.55 \\
\hline
\end{tabular}

Table S2. In all transient measurements the samples with a smaller device area $\left(1.68 \mathrm{~mm}^{2}\right)$ were used in order to improve the temporal resolution of electrical extraction. The samples with different areas $\left(1.68 \mathrm{~mm}^{2}\right.$ vs $\left.6.44 \mathrm{~mm}^{2}\right)$ are nominally identical. Similar OPV device characteristics were obtained by Tang et al. using various small-molecule donors. ${ }^{[7]}$ 


\section{Overview of simulation parameters}

\begin{tabular}{cccccccc}
\hline $\begin{array}{c}\alpha \text {-6T molar } \\
\text { fraction [\%] }\end{array}$ & $\begin{array}{c}\sigma_{\mathrm{h}} \\
\text { in } \mathrm{C}_{60} \\
{[\mathrm{meV}]}\end{array}$ & $\begin{array}{c}\sigma_{\mathrm{e}} \\
\text { in } \mathrm{C}_{60} \\
{[\mathrm{meV}]}\end{array}$ & $\begin{array}{c}\sigma_{\mathrm{h}} \\
\text { in } \alpha-6 \mathrm{~T} \\
{[\mathrm{meV}]}\end{array}$ & $\begin{array}{c}\sigma_{\mathrm{h} \mathrm{SCLC}} \\
\text { in } \alpha-6 \mathrm{~T} \\
{[\mathrm{meV}]}\end{array}$ & $\begin{array}{c}v_{\mathrm{h}} \\
\text { in } \alpha-6 \mathrm{~T}\left[\mathrm{~s}^{-1}\right]\end{array}$ & $\begin{array}{c}\Delta \mathrm{h} 1 \\
\text { scLC } \\
{[\mathrm{meV}]}\end{array}$ & $\begin{array}{c}\Delta_{\mathrm{h} 2 \text { SCLC }} \\
{[\mathrm{meV}]}\end{array}$ \\
\hline 1.5 & 61.97 & 58.48 & 196.80 & 144.30 & $2.2 \times 10^{11}$ & 0.048 & 0.170 \\
5.7 & 70.36 & 59.53 & 119.37 & 109.57 & $2.2 \times 10^{11}$ & 0.156 & 0.231 \\
10 & 77.51 & 62.48 & 113.15 & 108.42 & $2.2 \times 10^{11}$ & 0.066 & 0.143 \\
25 & 88.14 & 92.61 & 110.05 & 103.25 & $2.2 \times 10^{11}$ & 0.130 & 0.250 \\
50 & 100.27 & 98.55 & 117.70 & 101.07 & $2.2 \times 10^{11}$ & 0.086 & 0.163 \\
75 & - & - & - & 105.56 & $9.5 \times 10^{12}$ & 0.051 & 0.211 \\
\hline
\end{tabular}

\begin{tabular}{c|c}
\hline$a_{\mathrm{nn}}[\mathrm{nm}]$ & 1.8 \\
$v_{\mathrm{h}}$ in $\mathrm{C}_{60}\left[\mathrm{~s}^{-}\right.$ & $3.85 \times 10^{13}$ \\
$v_{\mathrm{e}}$ in $_{60}\left[\mathrm{~s}^{-1}\right]$ & $3.85 \times 10^{13}$ \\
$\begin{array}{c}\text { Langevin } \\
\text { prefactor }\end{array}$ & 1 \\
$\varepsilon$ & 3.6 \\
\hline
\end{tabular}

Table S3. Charge carrier hopping parameters were obtained from global least-squares fitting to transient experiments (by a single parameter set for all applied fields). The attempt-to-hop frequencies $v$ were first allowed to vary, but the resulting fit values of $v$ were found to be quite similar. We have thus fixed $v$ to its average value in subsequent fitting, allowing us to directly compare the obtained Gaussian disorder values $\sigma$ in the different materials. Parameters that were kept fixed during subsequent fitting are indicated by the grey area. Obtained energetic disorder $\sigma$ values from fitting the transient experiment were then used as the initial parameter guess for fitting the experimental steady-state hole-only SCLC data in Figure S5. We obtain reasonably similar disorder values when fitting the model to both transient ( $\sigma_{\mathrm{h}}$ in $\alpha-6 \mathrm{~T}$ ) and

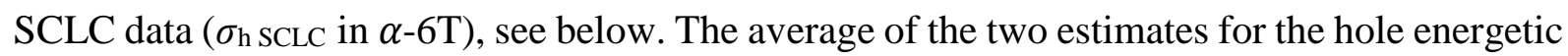
disorder $\sigma_{\mathrm{h}}$ in $\alpha$-6T was used in Figure 3a of the main text, where the standard error is indicated by the error bars. The rest of the parameters in the table are: $a_{\mathrm{nn}}$ is the lattice spacing, $\varepsilon$ is the dielectric constant, $\Delta$ is the injection barrier at the contact. 


\section{Fowler-Nordheim tunneling fit parameters}

\begin{tabular}{ccccccc}
\hline $\begin{array}{c}\alpha-6 \mathrm{~T} \text { molar } \\
\text { fraction [\%] }\end{array}$ & $P_{0}[\%]$ & $U_{0}[\mathrm{eV}]$ & $E[\mathrm{eV}]$ & $m^{*}$ & $d[\mathrm{~nm}]$ & $F=U / d$ \\
\hline 1.5 & 29 & 0.9 & 0.81 & $0.032 m_{\mathrm{e}}$ & 50 \\
5.7 & 35.9 & 0.9 & 0.75 & $0.032 m_{\mathrm{e}}$ & 50 \\
10 & 54.3 & 0.9 & 0.62 & $0.032 m_{\mathrm{e}}$ & 50 \\
25 & 27.8 & 0.9 & 0.66 & $0.032 m_{\mathrm{e}}$ & 50 \\
50 & 0 & 0.9 & - & $0.032 m_{\mathrm{e}}$ & 50 \\
\hline
\end{tabular}

Table S4. These parameters were used to fit the experimental data in Figure S6. The Fowler-Nordheim tunneling probability is reduced by the pre-factor $P_{0}$, which is also an estimate for the maximum percentage of holes that would undergo de-trapping from isolated $\alpha$-6T at an infinitely high electric field $F$. For a more detailed description of these parameters see Notes $\mathrm{S} 1$ and $\mathrm{S} 2$. 


\section{Supporting Notes}

\section{Fowler-Nordheim-type tunneling}

Note S1. The tunneling probability $P$ through a perfectly triangular energy barrier has been derived by Fowler and Nordheim: ${ }^{[8]}$

$P \cong P_{0} \exp \left[-4 \sqrt{2 m^{*}}\left(U_{0}-E\right)^{\frac{3}{2}} / 3 \hbar e F\right]$

Equation S1

Where $E$ is the energy of the particle, $U_{0}$ is the barrier height, $m *$ is the effective particle mass, $\hbar$ is the reduced Planck constant, $e$ is the elementary charge, $P_{0}$ is the scaling prefactor, $F$ is the electric field strength. Both $U_{0}$ and $E$ are schematically indicated in Figure S6, where it is also shown that an increase in particle energy $E$ eases tunneling through $U_{0}$. Increasing the electric field strength $F$ is expected to increase the tunneling probability by a factor of $P \propto \exp (-1 / F)$, as observed in our experiments (Figure S6).

Although the precise expression for the prefactor $P_{0}$ and the terms in the parenthesis is situation-specific, in most cases the Fowler-Nordheim tunneling equations retain their field-dependence as $P \propto \exp \left(-b h^{3 / 2} / F\right)$, where $b$ is the second Fowler-Nordheim constant and $h$ is the zero-field height of the barrier. This observation enables us to fit our experimental data by the use of Equation S1.

To make sure that Fowler-Nordheim tunneling fits to experiments are physically sound, we have fixed all known parameters in Equation S1 to experimental values. $U_{0}$ was set to the HOMO level difference between $\alpha-6 \mathrm{~T}$ and $\mathrm{C}_{60}(0.9 \mathrm{eV})$. The electric field strength $F$ was assumed to be zero at the experimentally determined built-in bias of $U_{\mathrm{bi}}=1 \mathrm{~V}$ (visible in Figure 3a of the main text), and otherwise equal to the effective applied bias $\left(U-U_{\mathrm{bi}}\right)$ divided by the sample thickness $d$. The effective mass of the tunneling hole $\left(m^{*}=0.032 m_{\mathrm{e}}\right)$ was determined using SCLC measurements as described in the Note S2 below. The effective mass of the hole was assumed to be the same for all donor concentrations. This approximation is valid below $10 \%$ molar of the donor where the $\mathrm{C}_{60}$ phase has not yet been significantly distorted (see Figure 4a of the main text). For the 25\% device, using the same effective mass may cause some error. However, our main goal here is to highlight the relevant physical mechanism, detailed derivation/analysis is outside the scope of this article.

We obtain good fits to experimental data, see Figure S6, strongly suggesting that the fast fraction of holes observed in experiment undergo Fowler-Nordheim-type field-assisted tunneling transfer from $\alpha$-6T and $\mathrm{C}_{60}$. Obtained fit parameters are summarized in the Table S4. Although the fit parameters should only be regarded as rough estimates, the fitted values for $U_{0}-E=0.09-0.28 \mathrm{eV}$ may appear surprisingly low given the fact that excitation takes place close to the center of the CT absorption manifold - a value closer to $U_{0}-E=0.9 \mathrm{eV}$ might be expected. We speculate that photo-induced hole transfer to $\mathrm{C}_{60}$ may occur before on-site thermalization, faster than $\sim 1 \mathrm{ps},{ }^{[9]}$ meaning that $\mathrm{E}>0$, effectively lowering the tunneling barrier. Moreover, the attractive Coulomb potential between the electron and the tunneling hole may 'round off' the triangular barrier (depending on the orientation of the CT pair), thus increasing the escape probability (similar to the known effect of the image force for field electron emission 
as described by Nordheim ${ }^{[10]}$ ). In either case, the fitted parameter values suggest that the photogenerated CT pairs are only weakly bound. 


\section{Tunneling through a rectangular barrier and the effective mass of the tunneling hole}

Note S2. Knowledge of the tunneling probability through a rectangular barrier and the hole localization length enable us to estimate the effective mass of the hole $m^{*}$, later used to fit the experimental field-dependence of hole de-trapping by Fowler-Nordheim-type tunneling (see Note S1 and Equation S1). The probability for tunneling through a rectangular barrier is:

$P=1 /\left[1+\frac{1}{4} \frac{U_{0}}{E\left(U_{0}-E\right)} \sinh ^{2}\left(\sqrt{\frac{2 m^{*} E\left(U_{0}-E\right)}{\hbar^{2}}} r\right)\right]$

Equation S2

For strongly attenuating barriers:

$\sqrt{\frac{2 m^{*} E\left(U_{0}-E\right)}{\hbar^{2}}} r \gg 1$

Equation S3

Equation S2 can be simplified to:

$P=\frac{E\left(U_{0}-E\right)}{U_{0}} \exp \left(-\frac{2}{\hbar} \sqrt{2 m^{*}\left(U_{0}-E\right)} r\right)$

Equation S4

Note that the probability for tunneling through a rectangular barrier is independent of applied bias and mainly limited by $U_{0}$ (donor and $\mathrm{C}_{60}$ HOMO level difference) and the barrier width $r$ (distance between donor sites). Therefore, hole tunneling can occur even at low fields relevant to operating OPV devices (short-circuit to $V_{\mathrm{OC}}$, i.e. applied voltage $0 \mathrm{~V}$ to $\approx 1 \mathrm{~V}$ ).

We relate the exponent of the tunneling probability in Equation S4 to experimentally measureable quantities by the commonly used equation for the charge carrier mobility:

$\mu=\mu_{0} r^{2} \exp (-2 \alpha r)$

Equation S5

In charge transport models $\alpha^{-1}$ is generally referred to as the localization length. By equating the exponential terms in Equations S4 and S5 we arrive at:

$\exp (-2 \alpha r)=\exp \left(-\frac{2}{\hbar} \sqrt{2 m^{*}\left(U_{0}-E\right)} r\right)$

Equation S6

$m^{*}=\hbar^{2} \alpha^{2} / 2\left(U_{0}-E\right)$

Equation S7

Given the above, a measurement of the charge carrier mobility versus donor site distance $r$ would give an estimate for the localization length $\alpha^{-1}$. Figure S9 shows that such measurements are indeed possible, allowing us to experimentally estimate the hole localization length to $\alpha^{-1}=1 \pm 0.1 \mathrm{~nm}$.

Then, substituting the experimental values of the HOMO level difference $\left(U_{0}-E=0.9 \mathrm{eV}\right)$ and the distance between isolated donor sites at homogenous dilution ( $<10 \%$ molar) from Table S1 into Equation S7, allows us to estimate the effective mass of the tunneling hole $\left(m^{*}=0.032 \pm 0.008 m_{\mathrm{e}}\right)$.

Note that contrary to the case of Fowler-Nordheim-type tunneling, where we have equated $U_{0}$ to the difference in the HOMO levels $\left(U_{0}=0.9 \mathrm{eV}\right)$, here, for the estimate of the effective mass in Equation S7 we have used $\left(U_{0}-E=0.9 \mathrm{eV}\right)$ instead, assuming $E=0$. This is because in the case of Fowler-Nordheim-type tunneling, most of the holes undergo field-assisted tunneling 
directly after being photo-generated (see Figure S7 for the fraction), and therefore may have excess energy that could reduce the tunneling barrier by $E$. In addition, Coulomb attraction may lower the triangular barrier further, as discussed in Note S1. In contrast, for the case discussed here, holes are expected to be trapped at the lowest energy of a neutral trap site $(E=0)$. This is in line with the significant difference in time scales: holes undergoing Fowler-Nordheim-type tunneling are extracted orders of magnitude faster than the slow fraction of holes that undergo direct tunneling, which is clearly visible in Figure 2 of the main text. Thus, compared to holes undergoing Fowler-Nordheim tunneling, the remaining slow fraction of holes are trapped in $\alpha$-6T for longer periods of time, enabling on-site relaxation to $E=0$. 


\section{Miller-Abrahams carrier hopping rate}

Note S3. The carrier hopping rate from a site with energy $E_{i}$ to a site with energy $E_{f}$, at an inter-site distance $r_{i f}$, is generally described by the Millar-Abrahams rate:

$v_{i f}= \begin{cases}v_{0} \exp \left(-2 \alpha r_{i f}\right) \exp \left(-\frac{E_{f}-E_{i}}{k_{\mathrm{B}} T}\right) & \text { if } \Delta E>0 \\ v_{0} \exp \left(-2 \alpha r_{i f}\right) & \text { if } \Delta E \leq 0\end{cases}$

Where $v_{0}$ is the attempt-to-hop frequency, $k_{\mathrm{B}}$ is the Boltzmann constant, $T$ is temperature.

In our model ${ }^{[11]}$ we implicitly account for the inter-site distance dependence via a simplified expression for the attempt-to-hop frequency $v$ :

$v=v_{0} \exp \left(-2 \alpha r_{i f}\right)$

Equation S9

According to Equation S8 with increasing inter-site distance $r_{\text {if }}$ the carrier hopping rate is expected to decrease exponentially, concomitantly $v$ in Equation S9 is also expected to decrease at the same rate. The inter-site distance is certainly larger for tunneling between isolated donor sites through several $\mathrm{C}_{60}$ molecules ( $\sim \mathrm{nm}$ ) as compared to tunneling between neighboring $\mathrm{C}_{60}$ $(\approx 1 \mathrm{~nm})$. We speculate that the observed 2 orders of magnitude difference between the carrier hopping frequencies obtained from our model fits to experiments for holes and electrons transported in $\mathrm{C}_{60}\left(v_{\mathrm{h}}=v_{\mathrm{e}}=3.85 \times 10^{13} \mathrm{~s}^{-1}\right)$ and holes tunneling between isolated donor sites $\left(v_{\mathrm{h}}=2.2 \times 10^{11} \mathrm{~s}^{-1}\right)$ may at least in part be attributed to the inter-site distance.

Using the same argument, the observed approximately 40-fold increase in SCLC hole mobility $\mu_{0}$ in $\alpha$-6T when the donor concentration reaches $75 \%$ (Figure S5), indicates a reduced inter-site distance: $v_{\mathrm{h}}$ increases approximately 40 -fold from $v_{\mathrm{h}}=2.2 \times 10^{11} \mathrm{~s}^{-1}$ to $v_{\mathrm{h}}=9.5 \times 10^{12} \mathrm{~s}^{-1}$ at $75 \%$ molar. Reduced inter-site distance is indicative of the formation of a continuous donor network. 


\section{References}

[1] W. F. Pasveer, J. Cottaar, C. Tanase, R. Coehoorn, P. A. Bobbert, P. W. M. Blom, D. M. de Leeuw, M. A. J. Michels, Phys. Rev. Lett. 2005, 94, 206601.

[2] J. M. Beebe, B. Kim, J. W. Gadzuk, C. Daniel Frisbie, J. G. Kushmerick, Phys. Rev. Lett. 2006, 97, 026801.

[3] S. Kazaoui, R. Ross, N. Minami, Phys. Rev. B 1995, 52, R11665.

[4] R. Coehoorn, W. F. Pasveer, P. A. Bobbert, M. A. J. Michels, Phys. Rev. B 2005, 72, 155206.

[5] W. D. Gill, J. Appl. Phys. 1972, 43, 5033.

[6] C. Tanase, E. J. Meijer, P. W. M. Blom, D. M. de Leeuw, Phys. Rev. Lett. 2003, 91, 216601.

[7] M. Zhang, H. Wang, H. Tian, Y. Geng, C. W. Tang, Adv. Mater. 2011, 23, 4960.

[8] R. H. Fowler, L. Nordheim, Proc. R. Soc. Lond. Math. Phys. Eng. Sci. 1928, 119, 173.

[9] P. A. Lane, P. D. Cunningham, J. S. Melinger, O. Esenturk, E. J. Heilweil, Nat. Commun. 2015, 6, 7558.

[10] L. W. Nordheim, Proc. R. Soc. Lond. Math. Phys. Eng. Sci. 1928, 121, 626.

[11] N. Felekidis, A. Melianas, M. Kemerink, Phys. Rev. B 2016, 94, 035205. 